\title{
Review-System-on-Chip SMO Gas Sensor Integration in Advanced CMOS Technology
}

\author{
Lado Filipovic $\oplus^{\mathrm{z}}$ and Ayoub Lahlalia $(\bullet$ \\ Institute for Microelectronics, Technische Universität Wien, E360, 1040 Vienna, Austria
}

\begin{abstract}
The growing demand for the integration of functionalities on a single device is peaking with the rise of IoT. We are near to having multiple sensors in portable and wearable technologies, made possible through integration of sensor fabrication with mature CMOS manufacturing. In this paper we address semiconductor metal oxide sensors, which have the potential to become a universal sensor since they can be used in many emerging applications. This review concentrates on the gas sensing capabilities of the sensor and summarizes achievements in modeling relevant materials and processes for these emerging devices. Recent advances in sensor fabrication and the modeling thereof are further discussed, followed by a description of the essential electro-thermal-mechanical analyses, employed to estimate the devices' mechanical reliability. We further address advances made in understanding the sensing layer, which can be modeled similar to a transistor, where instead of a gate contact, the ionosorped gas ions create a surface potential, changing the film's conduction. Due to the intricate nature of the porous sensing films and the reception-transduction mechanism, many added complexities must be addressed. The importance of a thorough understanding of the electro-thermal-mechanical problem and how it links to the operation of the sensing film is thereby highlighted.

(C) The Author(s) 2018. Published by ECS. This is an open access article distributed under the terms of the Creative Commons Attribution 4.0 License (CC BY, http://creativecommons.org/licenses/by/4.0/), which permits unrestricted reuse of the work in any medium, provided the original work is properly cited. [DOI: 10.1149/2.0731816jes]

Manuscript submitted August 10, 2018; revised manuscript received December 5, 2018. Published December 15, 2018. This was

Paper 1469 presented at the Seattle, Washington Meeting of the Society, May 13-17, 2018.

While aggressive device scaling has taken the front stage in the semiconductor industry for many decades, there is currently an everincreasing demand for functional integration in a single device. This means not only the integration of an increasing number of transistors along the path of Moore's Law, but also the integration of multiple applications on a single device, appropriately named More-than-Moore. The rise of the Internet of Things and the Internet of Everything are clear indicators of this trend. The first attempt at in-package integration dealt with connecting different dies with varying functionalities using bonding wires. However, this method can negatively impact performance and power dissipation, since long wires result in a high resistance/capacitance (RC) delays and an increased circuit resistance, limiting high frequency performance and reducing device lifetimes. The highest efficiency is reached when no bond wires are required and all functionalities are fabricated on a single substrate, deemed System-on-Chip (SoC).

The use of silicon as a substrate material for added functionality, including analog and radio frequency (RF) circuits, sensors and actuators, or biochips, allows for the efficient integration of microelectro-mechanical systems (MEMS) and complementary metal oxide semiconductor (CMOS) structures into a truly monolithic device. This is highly challenging, since the typically high temperatures associated with sensor fabrication has a negative influence on CMOS front end of line (FEOL) devices and back end of line (BEOL) metallization. However, the challenge is deemed well worth the effort since the integration of gas sensors with CMOS electronics is seen as a key enabler of smart gas sensors for mobile applications, allowing low power, low costs, and portability. ${ }^{1,2}$

This review discusses recent achievements in the integration of semiconductor metal oxide (SMO) MEMS gas sensors within an advanced CMOS technology, for which all fabrication steps, required for the sensor fabrication, are below $450^{\circ} \mathrm{C}$. The discussion is split into four main sections: The first one introduces semiconductor metal oxide sensors and their composition, while the second looks at the design of the microheater element, an essential component of the SMO gas sensor. The third section discusses the methods used to analyze the complex SMO structure, including the suspended membrane, while the final discussion looks at the modeling and simulation capabilities which have been developed in order to better analyze the sensing film itself. This type of in-depth analysis has enabled a significant improvement in the design and power optimization of advanced sensors and microheaters. Additionally, recent designs and models are presented and analyzed in this review. Before discussing the SMO sensor, the following subsection is meant to put the SMO sensor in context with other available gas sensor technologies. Its advantages over alternative solutions make it abundantly clear why these sensors are the subject of extensive research.

\section{Gas Sensing Mechanisms}

A large part of how we perceive the environment is shaped by the presence of various gases in our vicinity. As a natural sensor the human nose is able to detect hundreds of different odors, but it is not able to detect all harmful gases and fails absolutely when there is a need to detect specific gas concentrations. The ability to electrically detect our environment and the air we breathe has been a topic of extensive research over many decades. A wide range of applications and industries have a vested interest in gas sensor development including health and safety, ${ }^{3}$ automotive, ${ }^{4}$ environmental monitoring, ${ }^{5-7}$ and chemical warfare detection, ${ }^{8,9}$ among others. The feasibility to detect toxic and harmful gases in our environment through wrist watches, smart phones, tablets, and wearables is of particular interest, triggering substantial research. ${ }^{10-12}$ Furthermore, fabrication and process controls as well as laboratory analytics can be made more affordable with cheaper gas sensing equipment. Currently, a variety of gas sensing principles are being implemented in industry and research, e.g. semiconductor, optical, thermal conductivity, infrared (IR), quartz microbalance, catalytic, dielectric, electrochemical, and electrolyte sensors. ${ }^{13-15}$

Gas sensors can be classified as those whose sensing is based on a variation in electrical properties or variation in other properties. ${ }^{16}$ An excellent review of the different gas sensors is given by $\mathrm{G}$. Korotcenkov in Ref. 14, while A. Dey recently reviewed semiconductor metal oxide (SMO) gas sensors ${ }^{15}$ discussing the materials used, their sensitivity, selectivity, and stability. Dey's review thoroughly describes the characterization of the sensing material itself, including its response time, detection limits, and temperature of operation, while concentrating primarily on ammonia detection. The review of Korotcenkov deals primarily with identifying the different ways in which gas detection can be implemented and the advantages and disadvantages of those options. These are very clearly laid out in Ref. 14, which was also summarized more recently in Ref. 15 and enhanced and updated in this review, shown in Table I. The table includes the original characterizations by Korotcenkov from 2007, the update by Dey from 2018, as well as additions and updates for piezoelectric and photoionization sensors summarized from Refs. 17-19 and 20-23, respectively. This review concerns itself primarily with the CMOS 


\begin{tabular}{|c|c|c|c|c|c|c|c|}
\hline Parameter & SMO & Catalytic pellistor & Piezo-electric & Electro-chemical & Thermal pellistor & Photo-ionization & Infrared adsorption \\
\hline Sensitivity & E & G & E & G & $\mathrm{P}$ & E & $\mathrm{E}$ \\
\hline Accuracy & G & G & $\mathrm{E}$ & G & G & $\mathrm{E}$ & $\mathrm{E}$ \\
\hline Selectivity & $\mathrm{F}$ & $\mathrm{P}$ & $\mathrm{F}$ & G & $\mathrm{P}$ & $\mathrm{F}$ & E \\
\hline Response time & E & G & $\mathrm{E}$ & $\mathrm{F}$ & G & E & $\mathrm{F}$ \\
\hline Stability & G & G & G & $\mathrm{P}$ & G & E & G \\
\hline Durability & G & G & $\mathrm{F}$ & $\mathrm{F}$ & G & E & E \\
\hline Power & $\mathrm{E}$ & $\mathrm{E}$ & $\mathrm{F}$ & G & G & $\mathrm{P}$ & $\mathrm{F}$ \\
\hline Cost & $\mathrm{E}$ & $\mathrm{E}$ & G & G & G & $\mathrm{F}$ & $\mathrm{F}$ \\
\hline Footprint & $\mathrm{E}$ & G & G & $\mathrm{F}$ & G & $\mathrm{E}$ & $\mathrm{P}$ \\
\hline
\end{tabular}

integration, fabrication, and understanding the operation of SMO gas sensors. The sensor structure is discussed, including its fabrication and electro-thermal-mechanical operation. Subsequently, the recent advances in furthering our understanding of the sensing mechanism and its modeling are given.

Another significant advantage of SMO sensors is their reproducibility and repeatability. ${ }^{24}$ The ability to repeatedly fabricate the same structure with predictable operating conditions is essential when developing commercial devices. Without this, the industry would have never moved toward commercialization, since in mass production, high levels of certainty must exist when not every individual device can be precisely tested prior to its delivery to customer. While many other potential devices have been researched over the years, including graphene and other two-dimensional materials, these technologies are simply not mature enough to reach the reproducibility of SMO devices; this is why SMOs still dominate the semiconductor gas sensor market.

\section{Semiconductor Metal Oxide Sensors}

The detection in SMO sensors is based on changing electrical properties in the presence of a target gas. More specifically, the resistance of the film changes due to the interaction of gas molecules at its surface. From the summary of different gas sensors in use today given in Table I, it is clear why SMOs are currently the most popular choice, with its only flaw being selectivity, which is currently an active research field. In fact, similar to the piezoelectric sensor, the SMO's selectivity is primarily achieved using a sensor array, which allows for a simultaneous detection at multiple optimizations, such as at different operating temperatures or using different dopants. The collected sensor data can then be post-processed using a variety of methods to introduce selectivity.

The advantages and disadvantages of SMO alternatives are briefly mentioned here in order to convey to the reader the advantages that SMOs present over alternatives and that further work on improving the sensor's microheater as well as selectivity is well worth the effort. Even though there are several challenges, including the aforementioned selectivity and ensuring mechanically stable microheater integration, SMO sensors still provide meaningful advantages toward the development of integrated smart sensors.

The primary disadvantage of catalytic combustion is the lack of selectivity in the detection of a desired gas. Electrochemical sensors are not easily miniaturized to a portable size and suffer from poor stability, durability, and response time. Thermal conductivity sensors have very poor sensitivity and selectivity, while the power dissipation, portability, and cost trail behind those of an SMO sensor. Infrared (IR) absorption sensors have a very high selectivity and sensitivity, but are very difficult to be made portable with complex maintenance and high fabrication costs. If recent advances in the integration of IR sensors with silicon technologies prove to be fruitful, these hurdles may be overcome, resulting in a near ideal gas sensing device. ${ }^{25}$ In the meantime, the SMO sensor is one which has been embraced by industry and has been commercialized due to its high sensitivity, fast response time, low maintenance, cheap fabrication costs, and portability. ${ }^{26-31}$ Some questions still remain regarding the SMO's selectivity, stability, and durability, with room for improvement on several fronts.

The primary concerns for SMO sensors are:

- The functioning principle of the gas sensor is based on heating the metal oxide sensing layer to temperatures between $250^{\circ} \mathrm{C}$ and $500^{\circ} \mathrm{C}$ in order to provide enough energy for the necessary surface reactions to take place. Reducing the temperature to below $100^{\circ} \mathrm{C}$, while having a good sensing response, would lead to a reduction in the power consumption and improve the sensor reliability, since the associated thermal stress would be significantly reduced. One of the main research fields is, therefore, attempting to reduce the operating temperature of the sensing layer.

- The need for operation at high temperatures means that a microheater is required. Due to the required high temperatures, thermal isolation from the surrounding devices becomes essential, complicating the design and fabrication processes. This is also the reason why stability and durability should be improved to ensure long device lifetimes. In addition, since the provided temperature influences the sensing, knowing the exact microheater behavior is essential. This is not trivial and characterizing different types of sensors is a research study in itself.

- Another concern is with the SMO's selectivity, which is currently being addressed using sensor array structures. ${ }^{32-38}$ Sensors can be individually engineered to increase sensitivity toward a particular gas and when many sensors are combined, the collected data can be analyzed using post-processing techniques such as neural networks in order to better pinpoint which gas has adsorbed at the SMO surface. ${ }^{39}$ The required data analysis makes it even more attractive to integrate the sensor with digital CMOS circuits, since it allows for the post-processing to take place on the same chip, increasing speed and reducing losses associated with long interconnect lines.

- A thorough understanding of all the processes taking place during the SMO sensor's operation is not yet known. Recently it was shown that the sensing is not only due to a surface redox reaction with adsorbed oxygen, but that even in the absence of oxygen, a thin accumulation layer forms around the surface, thereby changing the film's resistivity and introducing a sensor signal. In addition, many studies have shown that the introduction of a dopant metal can improve the sensitivity and selectivity of SMO sensors. A model which includes all the effects of an additional dopant metal does not currently exist, while such a model would be beneficial in order to develop predictable models and a technology computer aided design (TCAD) environment for SMO sensor designs.

CMOS integration.-The semiconductor metal oxide sensor is on its way to becoming a universal sensor, since it can be used for many emerging applications in sensor networks, medical applications, food quality monitoring, and wearable devices. ${ }^{40,41}$ At the same time, the thin SMO film can detect a variety of gases, essential for measuring indoor and outdoor air pollution and toxicity in our environment, aspects of primary concert to our global health and safety. The discoveries which have enabled the integration of thin SMO films 


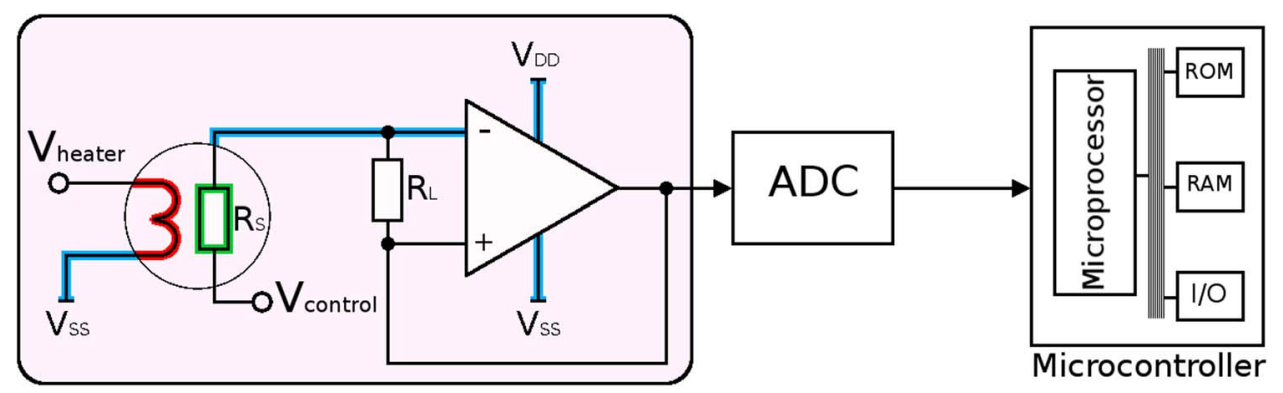

Figure 1. Typical schematic of a single SMO sensor with interface blocks. The sensor requires a heating element, a voltage follower, and an analog-to-digital converter (ADC). In order to analyze the obtained data, it is passed to a microcontroller, which is enabled with a read-only-memory (ROM), random access memory (RAM), and input/output (I/O) interfaces. ${ }^{42,43}$

within a CMOS fabrication sequence have opened up a world of possibilities for sensors integrated in electronic components and a broad integration of sensors in our daily lives, which is already packed with electronic components and devices. The CMOS integration has also made device production much more affordable than its alternatives.

A typical integrated resistive sensor circuit is shown in Figure 1. The complexity for its SoC integration is immediately evident: It requires a microheater, a sensing element, and analog and digital circuitry, all on a single chip. ${ }^{42,43}$ The colored sections in Figure 1 highlight elements, whose integration requires special attention:

- Green - The sensitive semiconductor metal oxide layer must be exposed to the ambient, thereby requiring to be deposited at the end of the CMOS sequence at low temperatures.

- Red - The microheater is required to heat the SMO layer to high temperatures, conducive to gas sensing. In this review we concentrate on the design and implementation of metallic microheaters in a suspended, full, or perforated membrane.

The integration between an SMO sensor and CMOS is twofold: First, CMOS electronics are required to enable the control of the voltage supplied to the heater to provide the increase in temperature, when operating in power-saving pulsed mode. In addition, the sensing signal itself can be processed using an analog/digital CMOS circuit, as shown in Figure 1. The second integration deals with the fabrication of the sensor devices. Allowing for the devices to be processed using a mature CMOS technology is essential to allow for highly costand power-efficient fabrication. Park et al. ${ }^{1}$ recently introduced an interface system between a metal oxide sensor and CMOS electronics, which is intended to provide a switching scheme for pulse width modulation to control the temperature of the heater and measure the sensor output simultaneously. These types of designs led to the development of fully CMOS integrated portable sensor modules for IoT applications. ${ }^{43}$

Sensor fabrication.-In Ref. 44, Lackner et al. show the importance of sensor integration with the CMOS fabrication technique, resulting in very low production costs and low power consumption. The crucial step is the integration of a microheater and suspended membrane. The microheater must be made of materials, preferably metals, readily available in a CMOS fabrication environment. The suspended membrane, which comprises the microheater and isolating layers, such as oxides and nitrides, also needs to be made possible within a CMOS fabrication technology, if the cost of the final device is to be minimized.

Another critical processing step, in addition to the fabrication of a suspended membrane, is the deposition of a metal oxide layer to act as the sensing element. The deposition of the sensing film itself can be incorporated with the CMOS fabrication sequence in one of several ways. These include sol-gel processing, ${ }^{45-48}$ chemical vapor deposition, ${ }^{49-51}$ sputtering, ${ }^{52-56}$ spray pyrolysis, ${ }^{57-63}$ pulsed-laser deposition, ${ }^{64}$ and rheotaxial growth and vacuum oxidation. ${ }^{65}$ Sputtering, chemical vapor deposition, and spray pyrolysis are quite straight forward to implement within a CMOS fabrication sequence, with spray pyrolysis being the most cost-effective option and the one in wide use today. ${ }^{57}$

The highest complexity in the fabrication of an SMO sensor is the inclusion of a microheater. The microheater requires thermal isolation from surrounding components, achieved with the formation of a suspended membrane, which uses air as a thermal insulator to the underlying silicon wafer, as shown in Figure 2. The formation of this membrane is the most challenging fabrication component, for which two main membrane types are currently being used: The closed membrane, which is etched from the back of the wafer, and the suspended membrane, which is etched from the front of the wafer. ${ }^{66,67}$ The two types are illustrated side by side in Figure 2a, where the top and side views of the two membrane types are shown. In Figure $2 b$, the side view of the active area is depicted in more detail, ${ }^{68}$ showing the effective air isolation below the membrane, while Figure $2 \mathrm{c}$ depicts the membrane materials, with a microheater sandwiched between isolation layers $\left(\mathrm{SiO}_{2}\right.$ or $\left.\mathrm{Si}_{3} \mathrm{~N}_{4}\right)$. In addition, the SMO film is shown, deposited on top of electrodes. However, it is also not uncommon to have electrodes deposited on top of the sensing film.

There are two main fabrication techniques for the membrane generation using front-side or back-side etching, which result in two membrane types, the suspended membrane or the closed membrane,

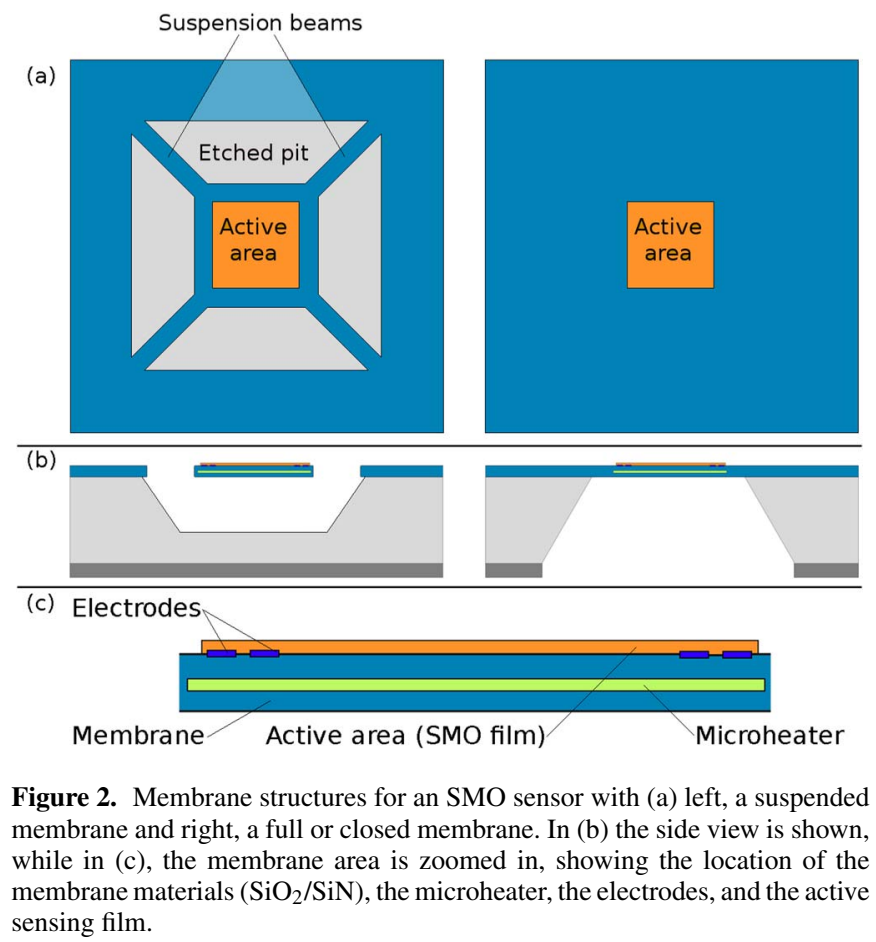


Front-side
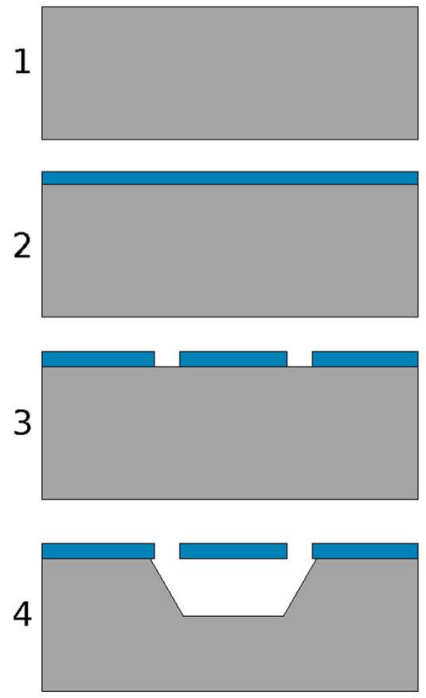

Membrane stack Silicon

Silicon dioxide Polyimide
Backside
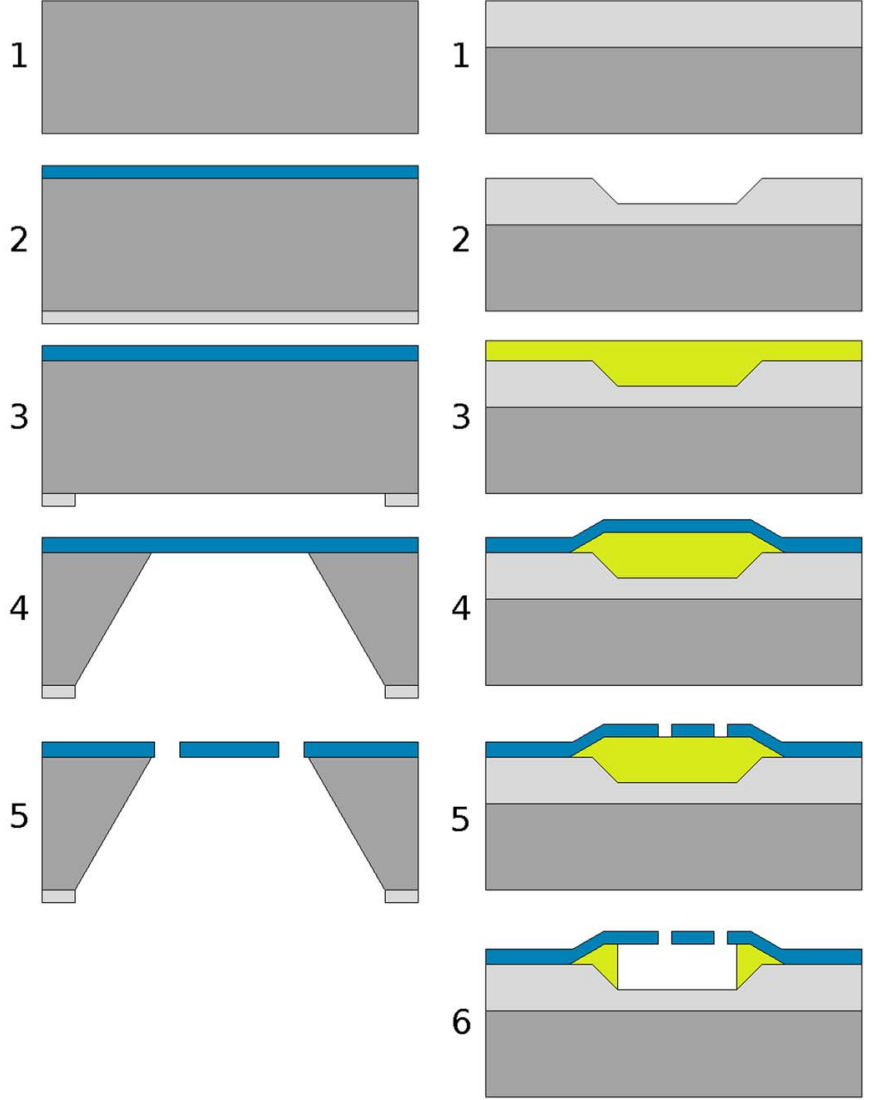

Figure 3. Etching techniques to release the MEMS membrane for SMO gas sensors. Front-side etching: (1) Start with a silicon wafer, (2) Deposit membrane layers, (3) Etch holes in the membrane to create the suspension beams, and (4) Apply wet chemical (or plasma) etching to create a hole below the membrane. Backside etching: (1) Start with a silicon wafer, (2) Deposit membrane layers and back side $\mathrm{SiO}_{2}$, (3) etch back side photoresist to open area below the sensor, and (4) Etch up to the membrane using wet chemical etching or DRIE, and (5) Optionally open the membrane from the top. Polyimide etching: (1) Start with themal oxide on silicon, (2) Etch a hole in the $\mathrm{SiO}_{2}$, (3) Deposit a layer of polyimide HD8820 whih fils the hole, (4) Deposit membrane layers, (5) Etch holes in the membrane, and (6) Selective plasma etches away polyimide through the holes, leaving the silicon and membrane materials in tact.

respectively, as depicted in the left and middle fabrication sequences in Figure 3. For front-side fabrication, there are two main methods to etch the hole, one of which is the use of highly selective wet chemical etchants such as potassium hydroxide $(\mathrm{KOH}),{ }^{69}$ ethylenediamine pyrocatechol (EDP), ${ }^{67}$ or tetramethylammonium hydroxide (TMAH) ${ }^{70}$ These etchants require the use of a silicon nitride or silicon oxide films as etch-stop layers. ${ }^{71}$ The wet chemical etching technique is very expensive and low-cost alternatives are sought after. The alternative low-cost option is the use of selective plasma etch processes, such as $\mathrm{SF}_{6}$-based plasma chemistries, often used for silicon and silicon dioxide etching. ${ }^{68,72}$ Although plasma etching is cheap and fully CMOS compatible, it is very difficult to avoid the lateral etching which takes place during this process. This is primarily because lateral etching is desired in one direction, in order to release the membrane, but not in the other, as it increases the size of the resulting air pocket. This process results in a very wide well, where more of the membrane is suspended than necessary. One way to deal with this is to introduce vertical nitride or oxide blocking layers, which would be costly to implement, or using a combination of back side etching with a final front-side etch of the membrane to create the suspension beams, as is represented visually in step 5 of the back side etch process given in Figure $3 .^{68,72}$

The air isolation below the active area can also be achieved by etching to the membrane from the back of the wafer, meaning that no suspension beams are required, but rather that a full membrane is used. This method, labeled as back side etching in Figure 3 (steps 14), although cheaper, has a reduced power efficiency, since heat is lost by thermal conduction and convection through the entire membrane, a principal heat loss component in these structures. In suspended membranes, the conduction only takes place through thin suspension beams. ${ }^{73}$ The back side etching can be performed using the same wet chemical etch techniques described for the front-side etching process or by using deep reactive ion etching (DRIE), which is cheaper, but more complex to implement. ${ }^{74}$ By adding an extra etching step form the front side, the closed membrane can be released to be identical to a suspended membrane. However, this requires non-trivial alignment between the front and back sides of the wafer.

More recently, STMicroelectronics has realized another method in generating the suspended membrane on a silicon wafer. ${ }^{75-77}$ First, an isolating silicon dioxide is deposited using thermal oxidation or chemical vapor deposition (CVD). A sacrificial Polyimide HD8820 is subsequently spin-coated and selectively etched to form the sensor cavity, as shown in the right side of Figure 3 and in Figure 4. The membrane material is composed of a Tantalum-Aluminum (TaAl) microheater, sandwiched between two silicon nitride $\left(\mathrm{Si}_{3} \mathrm{~N}_{4}\right)$ layers. $\mathrm{Si}_{3} \mathrm{~N}_{4}$ is deposited using low pressure chemical vapor deposition (LPCVD), while $\mathrm{TaAl}$ is patterned using physical vapor deposition (PVD) and subsequent plasma etching. The sensing material is deposited on top of the membrane, followed by the metal contacts. ${ }^{78}$ This novel technique allows for a fully front-side fabrication without the need for corrosive wet chemical etchants, while being compatible with CMOS fabrication. The applied sensing layer is tin dioxide $\left(\mathrm{SnO}_{2}\right)$, one of the most promising metal oxide materials for gas sensor applications. As mentioned previously, $\mathrm{SnO}_{2}$ can be deposited using a variety of 


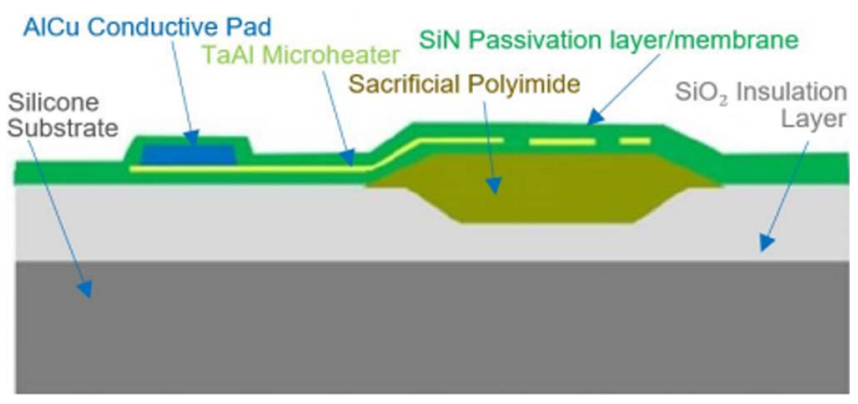

Figure 4. Two-dimensional cross-section cut through the material stack making up the microheater. ${ }^{77}$ The primary materials are shown, including the sacrificial Polyimide, which, when removed, forms the air cavity and the suspended membrane, ensuring thermal isolation between the membrane microheater and the underlying silicon wafer.

techniques: CVD, sputtering, pulsed-laser deposition, sol-gel process, and spray pyrolysis. The choice of sensing material is discussed in more detail in the following section.

Choice of sensing film.-The miniaturization of transistors and other electronic devices has proven to be essential in advancing our technological capabilities. However, the chemical sensor field, until recently, lagged behind the overall progress of CMOS devices. Discoveries in the application of metal oxide semiconductors have narrowed this somewhat by enabling sensor miniaturization and integration with other electronics. In the early 1950s Brattain and Bardeen ${ }^{79}$ demonstrated for the first time that several semiconducting materials display a sensitivity toward the presence of gas molecules, especially when heated to high temperatures. The conductivity of these materials changed when the chemical composition of the ambient gas changes. Following this discovery, in the early 1960s, the first gas sensing device based on a thin zinc oxide $(\mathrm{ZnO})$ film was proposed, operating at $485^{\circ} \mathrm{C} .^{80}$ By 1967 , Shaver showed a new method to improve the material's sensitivity by adding small amounts of noble metal dopants such as platinum, rhodium, iridium, gold, and palladium. ${ }^{81}$

Researchers have since intensified the search for new gas sensing materials and the combinations of dopants which can improve their sensitivity and selectivity. Many SMOs have been extensively studied for their gas sensing properties, including indium oxide $\left(\mathrm{In}_{2} \mathrm{O}_{3}\right)$, indium-tin oxide (ITO), cadmium oxide (CdO), zinc tin oxide $\left(\mathrm{ZnSnO}_{4}\right)$, lead oxide $(\mathrm{PbO})$, and many more. Among the most promising, and those now beginning to enter commercialization, are tin oxide $\left(\mathrm{SnO}_{2}\right)$, zinc oxide $(\mathrm{ZnO})$, and tungsten trioxide $\left(\mathrm{WO}_{3}\right)$. These three SMOs fulfill most, if not all, of the requirements for a good gas sensing performance, which is their sensitivity to a broad spectrum of potentially harmful gases, ease of deposition, and low cost of fabrication. ${ }^{13}$ The first SMO based gas sensing device was patented by Taguchi and it was dedicated to safety monitoring using a porous tin oxide $\left(\mathrm{SnO}_{2}\right)$ film with a palladium doping. ${ }^{82,83}$ Since then, a plethora of research has been centered around finding the perfect metal oxide material for specific gas sensing capabilities. Recently, it has become clear that $\mathrm{SnO}_{2}$ is likely the best metal oxide for gas sensors due to its ability to detect almost all relevant gases. ${ }^{13,84}$

The gas sensing capability of $\mathrm{SnO}_{2}$ is well known and, over the last few years, it has become the most commonly used SMO material for gas sensing, resulting in its commercialization. ${ }^{26-31} \mathrm{SnO}_{2}$ is also the material which was shown to be the easiest to integrate into the CMOS silicon technology. ${ }^{13,44,74,85}$ Figure 5a shows the response of an $\mathrm{SnO}_{2}$ thin film to the presence of carbon monoxide (CO) in the environment, compiled from recent publications from A. Köck et al. ${ }^{44,86}$ Here, and in subsequent figures, the sensitivity is represented as the ratio, in percent, of the resistance reduction when in the presence of a target gas, compared to the resistance in air or inert ambient, given by

$$
\text { Sensitivity }=\frac{R_{\text {air }}-R_{\text {gas }}}{R_{\text {air }}} \cdot 100 \%,
$$

where $R_{\text {air }}$ represents the baseline resistance after stabilization in ambient air and $\mathrm{R}_{\mathrm{gas}}$ is the resistance of the sensitive layer after exposure to a target gas mixed in air. The response to the presence of $\mathrm{CO}$ shown in Figure 5a has been measured at several temperatures for a spray pyrolysis deposited film with a thickness of $50 \mathrm{~nm}$. The authors note that the addition of a $2 \mathrm{~nm}$ evaporated gold ( $\mathrm{Au}$ ) film results in a significant improvement in the sensor response. For a $50 \mathrm{~nm}$ thin film, varying the temperature from $350^{\circ} \mathrm{C}$ to $400^{\circ} \mathrm{C}$ did not greatly influence the response.

The $\mathrm{CO}$ response shown in Figure $5 \mathrm{~b}$ has been measured at $300^{\circ} \mathrm{C}$ for a spray deposited film, which also includes impurities in the form of platinum (Pt) nanoparticles. ${ }^{87}$ The presence of $\mathrm{Pt}$ impurities results in a significant increase in the sensing performance, but only when a very small amount of $\mathrm{Pt}(0.2 \mathrm{wt} \%)$ is present. When the $\mathrm{Pt}$ concentration increased to $2 \mathrm{wt} \%$, the sensing response is actually reduced. While no accepted physical explanation exists for this phenomenon, the presence of Pt increases the sensitivity due to the dissociation of oxygen on platinum. Then, the activated oxygen species reach the $\mathrm{SnO}_{2}$, where they finally react with $\mathrm{CO} .{ }^{87}$ The low signal when $2 \mathrm{wt} \%$ $\mathrm{Pt}$ is used may lead to "localized" CO consumption without electron transfer, resulting in no changes in the $\mathrm{SnO}_{2}$ film's resistivity. ${ }^{87}$ More discussion on this topic is given in the final section, dealing with surface chemisorption of noble metal doped SMO films. The symbols in the figure represent measured data while the solid lines are best-fit power-law lines, discussed in more detail in Ref. 88.

The influence of metal additives to improve selectivity was recently further addressed and tested by Tangirala et al. in Ref. 89 . The influence of copper $(\mathrm{Cu})$, platinum $(\mathrm{Pt})$, and palladium $(\mathrm{Pd})$ doped $\mathrm{SnO}_{2}$ on $\mathrm{CO}$ sensing was studied. The authors proposed incorporating metal dopants with chemical and impregnation methods by using urea and ammonia as precipitating agents. They found that the highest sensitivity was achieved when doping was incorporated using chemical methods with urea precipitator, while $\mathrm{Cu}: \mathrm{SnO}_{2}$ provided enhanced sensitivity, when compared to $\mathrm{Pt}$ or $\mathrm{Pd}{ }^{89}$ shown in Figure $5 \mathrm{c}$. The improvement achieved using a urea precipitator was attributed to this method providing uniform and homogeneous nanoparticles during synthesis, while the high response of $\mathrm{Cu}: \mathrm{SnO}_{2}$ is due to the excellent association and dissociation of oxygen $(\mathrm{O})$ in the presence of $\mathrm{CO}$ at the sensor operating temperature to form $\mathrm{CuO}^{89}$

\section{Microheater Design}

As previously mentioned, the microheater is one of the key components of the SMO gas sensor since the sensing film is only activated at elevated temperatures. The choice of microheater and membrane materials and microheater geometry is essential in enabling a uniform temperature distribution across the active sensor region and ensuring a minimal power dissipation, since the heater is also the sensor's most power-hungry component. The heater must be able to provide a predictable temperature, so that the power/temperature relationship can be appropriately characterized. Otherwise, if the temperature delivered is not the one expected, the operation of the sensing element will be incorrect.

Microheater material.-Different materials have been used for the microheater, including silicon carbide $(\mathrm{SiC}),{ }^{90}$ polysilicon, ${ }^{91,92}$ molybdenum, ${ }^{93,94}$ platinum, ${ }^{95}$ and tungsten. ${ }^{96}$ The membrane, which surrounds the microheater, is usually comprised of some combination of silicon dioxide $\left(\mathrm{SiO}_{2}\right)$ and silicon nitride $\left(\mathrm{Si}_{3} \mathrm{~N}_{4}\right) .^{70,78,97}$ The membrane is important in providing a platform on which the microheater and sensing film are suspended. Recent interest in $\mathrm{SiC}$ based microheaters stray from the typical $\mathrm{SiO}_{2} / \mathrm{Si}_{3} \mathrm{~N}_{4}$ stack, but their fabrication is very complex and thereby also cost intensive. ${ }^{90}$ The $\mathrm{SiC}$ microheaters are also not compatible with CMOS fabrication and therefore will not be covered here. 


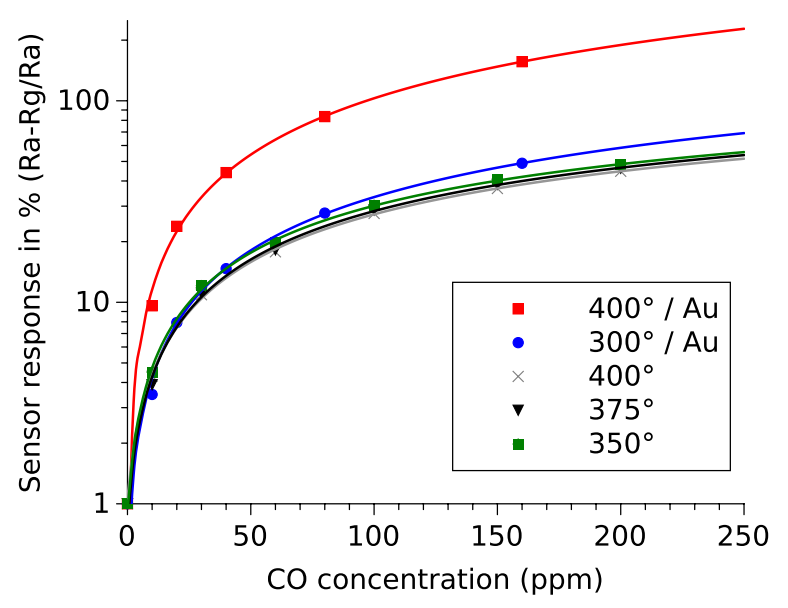

(a)

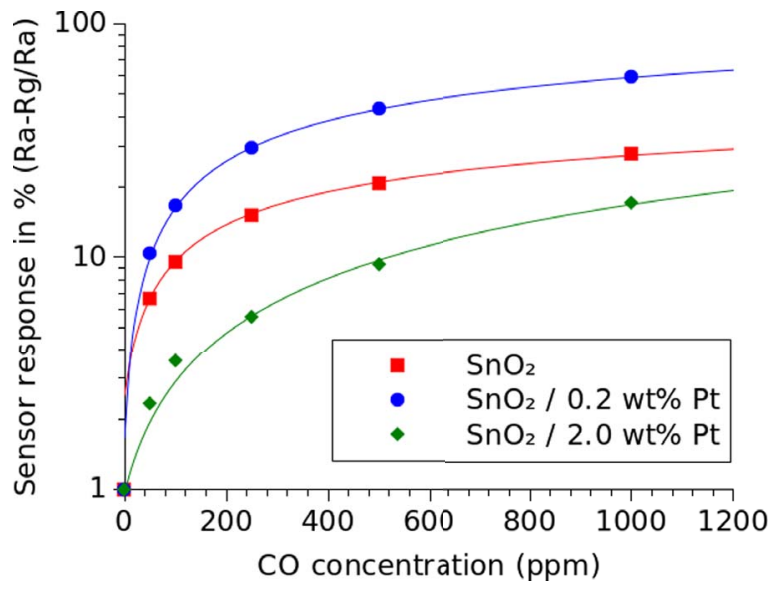

(b)

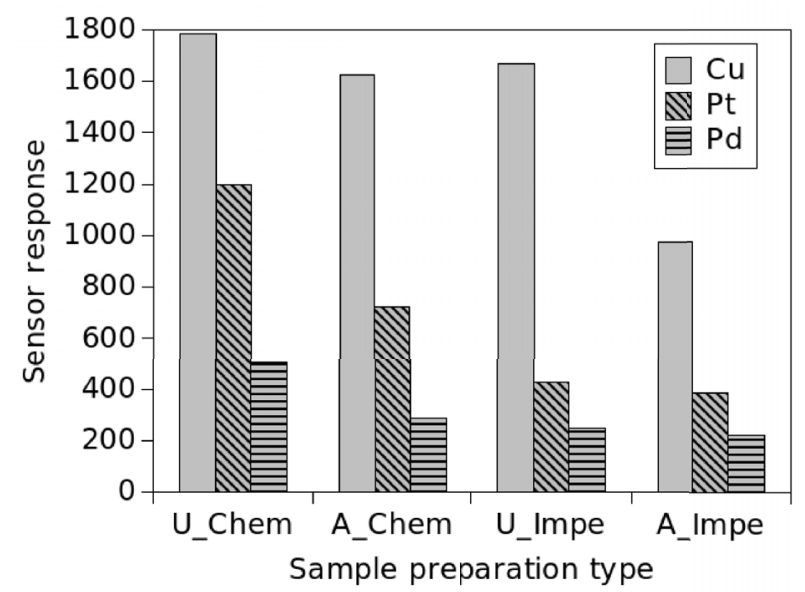

(c)

Figure 5. $\mathrm{SnO}_{2}$ thin film sensor response during exposure to a varying concentration of carbon monoxide (CO) gas. In (a) Köck et al. ${ }^{44,86}$ showed the influence of temperature and evaporated gold particles on the sensor response on a $50 \mathrm{~nm}$ thin spray deposited film. In (b) the Mädler et al ${ }^{87}$ discussed the influence of platinum (Pt) doping on a spray deposited $\mathrm{SnO}_{2}$ film. In (c) V. K. K Tangirala et al. ${ }^{89}$ discussed the influence of various dopants, doping methods, and precipitating agents in the sensitivity of an $\mathrm{SnO}_{2}$ film toward $\mathrm{CO}$. The temperatures used during the measurements correspond to the optimal temperature of operation for the detection of the target gas. $\mathrm{Ra}$ and $\mathrm{Rg}$ are the film resistances in air and in the presence of a target gas, respectively.

In the early years of micro-hotplate development, the frequently used materials were those readily available in a CMOS fabrication facility, including polysilicon, aluminum, and gold. ${ }^{98,99}$ Eventually it was found that these materials are not ideal since they suffer from electromigration defects and have poor contact properties. Platinum is frequently used as a heating element today due to its ability to deal with high current densities and it being chemically inert at high temperatures. ${ }^{66}$ The main drawback of platinum is its cost and it having a positive temperature coefficient of resistance (TCR), which magnifies hotspot effects, leading to concerns over the long-term reliability of the microheater and potential response drift. ${ }^{100}$ Tungsten was also suggested as a potential material, ${ }^{74}$ and it seems ideal since it is effectively resistant to electromigration, but its tendency to form an oxide at temperatures above $300^{\circ} \mathrm{C}$ makes it problematic for use as a heating element. Currently, research into nickel and nickel alloys for micro-heaters is intensified ${ }^{67,101-103}$ due to their low coefficient of thermal expansion (CTE), resistance to humidity, and high Young's modulus. Tantalum-Aluminum ( $\mathrm{TaAl})$ is another promising composite material, recently suggested in Ref. 97 , the advantage of which is its ability to maintain mechanical strength at high temperature and the negative TCR of about $-100 \mathrm{ppm} /{ }^{\circ} \mathrm{C}$.

A good microheater material is characterized with having a low thermal conductivity, high melting point, high electrical resistivity, low fabrication costs, low CTE, low Poisson's ratio, and most importantly, high compatibility with MEMS and CMOS fabrication techniques. ${ }^{66}$

Microheater geometry.-Many attempts have been made over the years to optimize the geometry of the heater in order to achieve temperature uniformity in the active membrane region. Several designs involve the placement of a highly thermally conductive element (silicon, polysilicon, or metal) below or above the microheater in order to distribute the heat more uniformly. ${ }^{1}$ However, this method results in additional lithography steps and increased fabrication costs. An additional and widely used approach is the efficient modulation of the microheater geometry. The geometries can be broadly classified as rectangular, square, circular, or irregular, further subdivided into honeycomb, drive wheel, elliptical, etc. shown in Figure 6 and characterized by several research groups. ${ }^{76,104,105}$ The meander design, shown in Figure 7, is most commonly used in combination with rectangular or circular geometries. The line widths and the separation between lines has a significant influence on the efficiency of the temperature distribution. Minimizing the separation, or pitch, can lead to an improved power uniformity and lower power dissipation.

The microheater geometry plays an integral role in defining the sensor performance. The quality of the microheater to provide a 


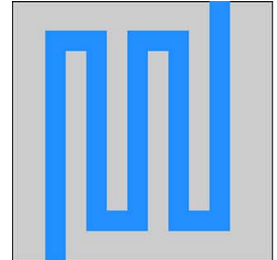

(a)

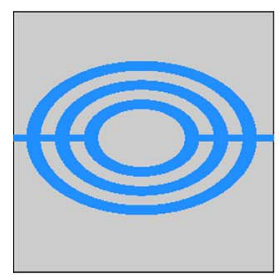

(g)

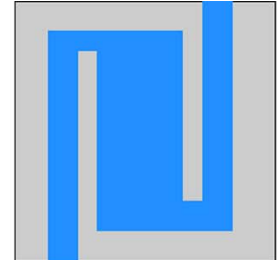

(b)

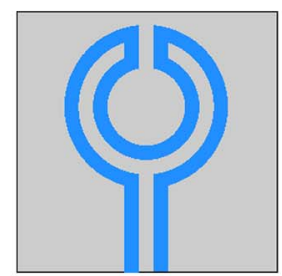

(h)

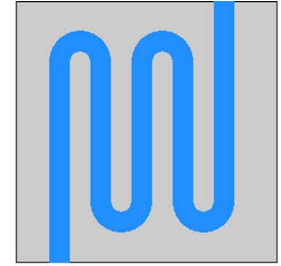

(c)

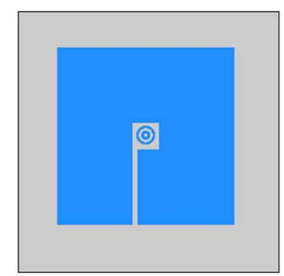

(i)

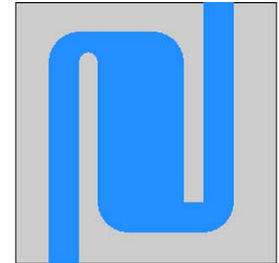

(d)

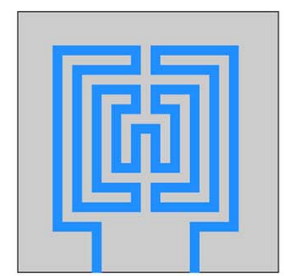

(j)

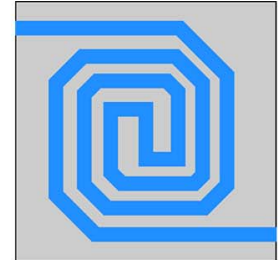

(e)

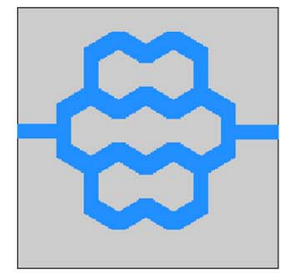

(k)

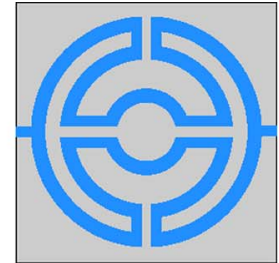

(f)

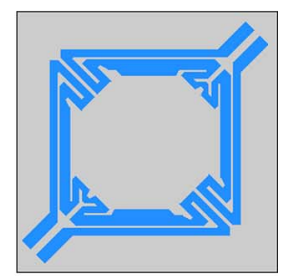

(I)

Figure 6. Microheater geometries characterized and modeled by different research groups. ${ }^{76,104,105}$ These include several shapes: (a) Meander, (b) S-meander, (c) Curved, (d) S-curved, (e) Double spiral, (f) Drive wheel, (g) Elliptical, (h) Circular, (i) Plane plate, (j) Fin shape, (k) Honeycomb, and (l) Irregular.

predictable temperature uniformly across the entire active sensor area is essential to provide confidence in the sensor's response. However, increased intricacies in the geometries and attempts to reduce the pitch mean an increased complexity in the fabrication technique and potential reliability concerns. Having many corners and sharp turns for the current could result in current crowding and increase the likelihood of early electromigration failure, cracking, and localized deformations. Circular structures and rounded corners are therefore generally preferred as an alternative to the typical rectangular meander shape from Figure 7.

Power efficiency.-An extensive amount of work has recently been devoted to novel microheater designs to improve the temperature uniformity, power consumption, and thermal isolation, while easing the fabrication requirements. The relationship between the input power and microheater temperature is near linear, allowing for the use of a microheater efficiency parameter in terms of ${ }^{\circ} \mathrm{C} / \mathrm{mW}$. However, this is insufficient when trying to characterize microheaters across a broad range of sizes, since the heater area plays a significant role in the power dissipation. Therefore, in this review we treat power efficiency as the temperature increase when $1 \mathrm{~mW}$ of power is applied to a microheater with equivalent area of $1 \mathrm{~mm}^{2}$, in terms of $\mathrm{mm}^{2} \cdot \mathrm{K} / \mathrm{mW}$. The efficiency for recently published microheaters since 2000 is summarized in Table II. In this review we concentrate primarily on CMOS-integrable designs. The summary is sorted by year of publication, ranging from 2000 until $2018 .^{73,74,78,91,100,106-121}$

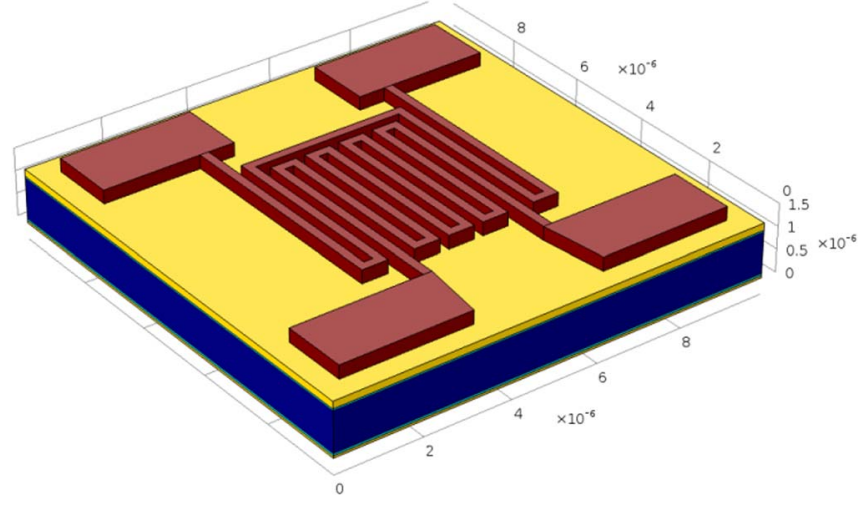

Figure 7. Meander microheater design with a square pattern. The second line is used for temperature measurement.
With regard to Table II, it should be noted that in Ref. 119 the values used were only mentioned as typical values for power consumption of micro-hotplates using an estimated efficiency of $15^{\circ} \mathrm{C} / \mathrm{mW}$, which was then applied to their structure's geometry to find the power dissipation. It is not clear what was precisely measured or simulated to obtain this number. In some of the studies mentioned, the active area covers a very small section of the microheater; this is not uncommon since temperature uniformity is almost impossible to achieve up to the edge of the microheater. However, in Ali et al. ${ }^{74}$ the active area covers only $7 \%$ and $0.6 \%$ of the membrane area for the $26 \mathrm{~mW}$ and $11 \mathrm{~mW}$ heaters, respectively. This can help improve the power efficiency numbers since the rest of the heater can have very poor uniformity or be thermally isolated, while only the small active area is exposed, explaining their very good power efficiency. At first sight it may seem that a better efficiency evaluation should be dependent on the required active area. However, our aim is to evaluate the microheater design itself and not the size of the sensor it is heating. Also, introducing this metric would then force the introduction of other factors including temperature uniformity, fabrication complexity, microheater and membrane thicknesses, among many others. Our goal in this review was to summarize the efficiency of the complete microheater design and heater area purely concerning the power-temperature relationship, irrespective of the active area or performance factors. It is clear that having an efficiency metric, which takes all components into account, would be desirable to have in the future.

From Table II we further extract a general trend that larger microheaters have a higher value for the power efficiency. This is most evident in the works of Siegele et al. ${ }^{118}$ and Ali et al. ${ }^{74}$ In these works, the authors fabricated two microheaters each using the identical fabrication process, but with different surface areas. This resulted in the larger heater requiring more power, but in it having a better power efficiency. A possible explanation for this is the fact that heaters with a larger surface area have a lower ratio of circumference to surface. Since the presented heaters have a closed membrane, much of the heat is lost on the sides, or along the circumference of the microheater. This can explain the trend of improved power efficiency with increasing size. In Ali et al. ${ }^{74}$ the larger microheater has a circumference which is approximately $46 \%$ wider than that of the smaller design, resulting in an efficiency improvement of about $32 \%$. Similarly, the results from Siegele et al. ${ }^{118}$ suggest an increase in the side length of a square heater by $30 \%$ results in an improvement of the power efficiency by about $23 \%$. There are clearly additional effects which need to be considered to properly evaluate a microheater, but these works validate the general hypothesis that fewer larger microheaters are preferred to many smaller ones. In the context of a sensor array, shown in Figure 8, it would be much more efficient if the sensors did not each have an 
Table II. Characteristics and efficiency of recently-published microheaters. The power efficiency is calculated as the temperature increase above room temperature, in Kelvin, over a $1 \mathrm{~mm}^{2}$ area when $1 \mathrm{~mW}$ is applied to the microheater $\left(\mathrm{mm}^{2} \cdot \mathrm{K} / \mathrm{mW}\right)$.

\begin{tabular}{|c|c|c|c|c|c|c|}
\hline Area x1000 $\left(\mathrm{um}^{2}\right)$ & Heater material & Microheater type & Temperature $\left({ }^{\circ} \mathrm{C}\right)$ & Power $(\mathrm{mW})$ & Efficiency $\left(\mathrm{mm}^{2} \cdot \mathrm{K} / \mathrm{mW}\right)$ & Ref. \\
\hline 22.5 & Polysilicon & S-Shaped & 420 & 64.5 & $0.14^{*}$ & 106 \\
\hline 90 & Polysilicon & MOSFET & 300 & 100 & $0.25^{*}$ & 107 \\
\hline 10 & Polysilicon & Meander & 300 & 27.5 & 0.10 & 91 \\
\hline 562.5 & Platinum & Meander & 300 & 75 & 2.10 & 108 \\
\hline 1.6 & Platinum & Meander & 400 & 9 & 0.07 & 109 \\
\hline 10 & Platinum & Meander & 600 & 33 & 0.18 & 11 \\
\hline 70.8 & Polysilicon & Circular & 300 & 50 & 0.40 & 11 \\
\hline 58.1 & Platinum & Circular & 350 & 190 & 0.10 & 11 \\
\hline 246 & Tungsten & Circular & 500 & 26 & $4.55^{+}$ & 74 \\
\hline 70.8 & Tungsten & Circular & 500 & 11 & $3.09^{+}$ & 74 \\
\hline 4000 & Dilver P1 & Meander & 200 & 130 & 5.54 & 115 \\
\hline 10 & Polysilicon & Meander & 400 & 10 & 0.38 & 11 \\
\hline 14.6 & Platinum & Meander & 400 & 18 & 0.31 & 10 \\
\hline 283 & Platinum paste & Circular & 600 & 35 & $4.69^{\ddagger}$ & 119 \\
\hline 2.5 & Platinum & Meander & 400 & 11.8 & 0.08 & 73 \\
\hline 283 & Silicon & Circular & 660 & 83 & 2.18 & 120 \\
\hline 10 & Tungsten & Meander & 480 & 20.1 & $0.23^{*}$ & 121 \\
\hline 25.3 & Platinum & Circular & 300 & 6.8 & $1.04^{*}$ & 78 \\
\hline
\end{tabular}

*Simulation-based studies;

${ }^{\ddagger}$ Mentioned in Ref. 119 as typical values, assuming an efficiency of $15^{\circ} \mathrm{C} / \mathrm{mW}$;

${ }^{+}$The active area covers only $7 \%$ and $0.6 \%$ of the membrane area for the $26 \mathrm{~mW}$ and $11 \mathrm{~mW}$ heaters, respectively.

individual microheater, but if one large microheater could be engineered to serve multiple sensing films. A design which takes this into consideration is discussed in the next section.

Recent microheater designs.-The recent designs achieved by Lahlalia et al. in Ref. 78 have an area of $0.9 \mathrm{~mm} \times 0.6 \mathrm{~mm}$, with a structural membrane stack formed with layers of 500nm thick silicon dioxide $\left(\mathrm{SiO}_{2}\right), 300 \mathrm{~nm}$ thick silicon nitride $\left(\mathrm{Si}_{3} \mathrm{~N}_{4}\right)$, and an additional $500 \mathrm{~nm}$ thick $\mathrm{SiO}_{2}$. Platinum was used for the microheater and electrodes for its stability, linearity, and resistance to oxidation at a broad range of operating temperatures. Aluminum-copper $(\mathrm{AlCu})$ is chosen for the microheater pads for its high electrical conductivity and low thermal conductivity. For electrical insulation between microheater and sensor contact metallization, a 300nm thick silicon dioxide layer is deposited on top of the microheater. The two recently suggested novel geometries, which address key concerns for gas sensor developers are the microheater array design and the dual hotplate design, shown in Figure 9. A short description of the designs will be given here, while an in-depth analysis is provided in Ref. 78. The principal goals of the designs are to provide a power-efficient sensor array and to enable a uniform temperature distribution across the active sensor

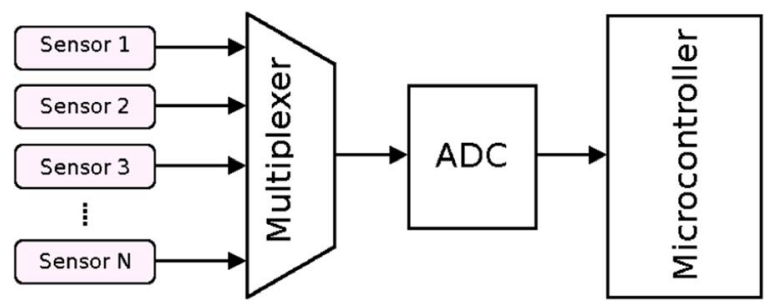

Figure 8. Sensor unit showing a sensor array with interface electronics blocks. A sensor array allows for selectivity improvements, but requires multiple sensors to read the gates in the air at the same time. area without increased fabrication complexity, otherwise introduced by a heat spreading plate. By concentrating on these two properties, the designs describe the general trends of significant interest to sensor manufacturers: miniaturization, power efficiency, and improved selectivity.

Microheater array.-The microheater array design is shown in Figure 9a and combines small resistances in an array instead of a conventional single-layer microheater. This allows for the localized heating of the sensing layer to different temperateness at different locations, thereby allowing for a natural integration with a sensor array using a single heat source and a reduced power consumption. The small resistances also provide an ultra-fast thermal response time allowing the microheater, which can operate in ultra-short pulse mode, to further reduce the average power consumption to a few hundred $\mu \mathrm{W} .^{1}$

Since the sensitivity of the SMO films toward target gases is optimal only at a single temperature, as depicted in Figure 10, the array can be used to simultaneously detect reactions at multiple temperatures. Here, we see the sensitivity of doped and undoped $\mathrm{SnO}_{2}$ thin films in detecting methane $\left(\mathrm{CH}_{4}\right), \mathrm{H}_{2}, \mathrm{CO}$, propane $\left(\mathrm{C}_{3} \mathrm{H}_{8}\right)$, isobutene (i- $\left.\mathrm{C}_{4} \mathrm{H}_{10}\right)$, ethanol $\left(\mathrm{C}_{2} \mathrm{H}_{5} \mathrm{OH}\right)$, and nitric oxide (NO) from several published works on $\mathrm{SnO}_{2}$ sensors. ${ }^{122-124}$ The collected measurements can subsequently be processed and treated using non-parametric analysis such as principal component analysis, discriminant functions, or neural networks to distinguish between each gas, improving the overall selectivity of the SMO sensor. ${ }^{125,126}$ Recently, an additional improvement on this design was suggested by introducing two sensing layers, deposited within the same membrane structure. ${ }^{78}$ The design allows for further potential in adjusting the microheater dimensions and position in order to incorporate even more sensing layers, effectively addressing the problem of selectivity in SMO gas sensors. With this design, a reduction in current crowding by about $20 \%$ was noted, when compared to conventional designs. 


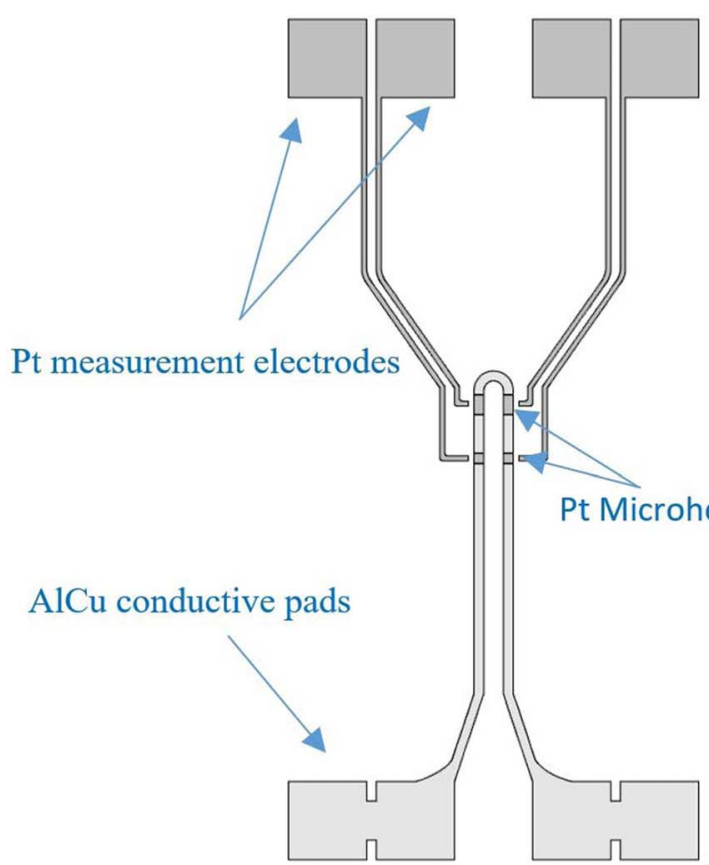

(a)

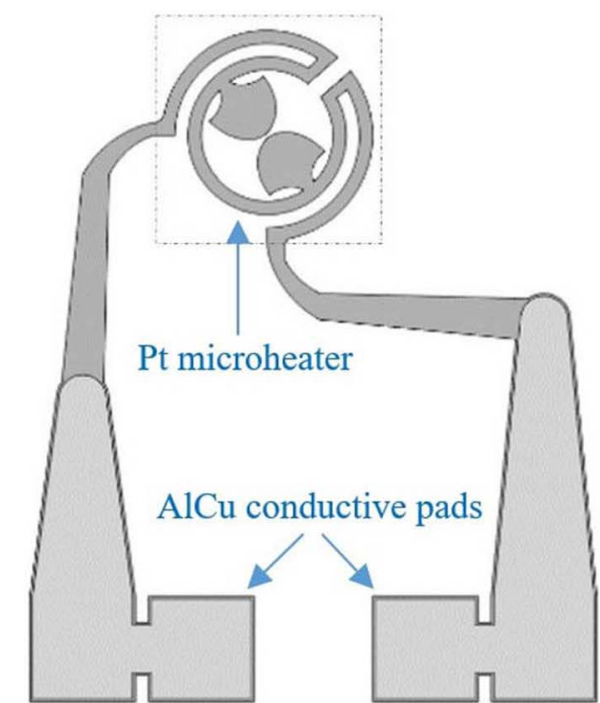

(b)

Figure 9. Novel microheater designs, including (a) microheater array and (b) dual hotplate geometries.

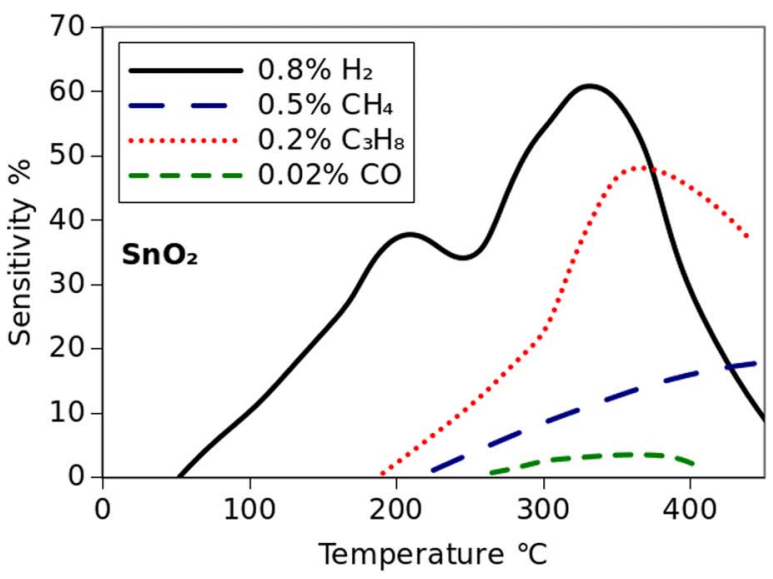

(a)

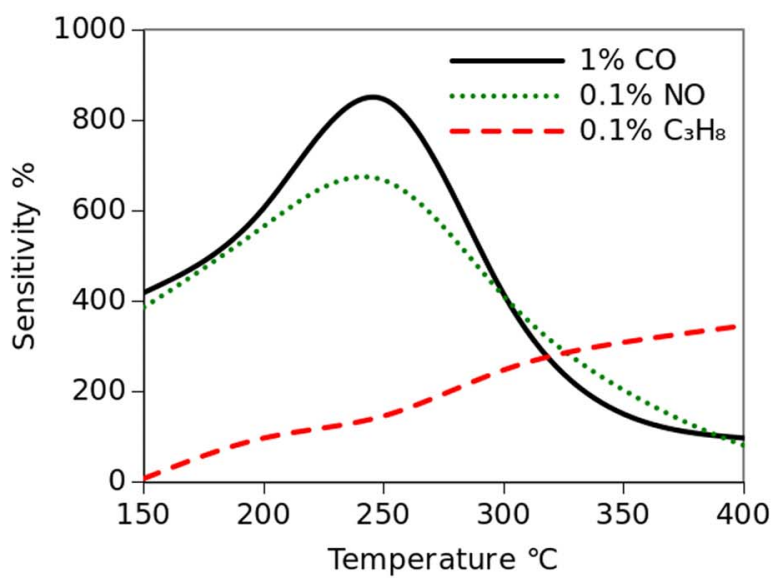

(c)

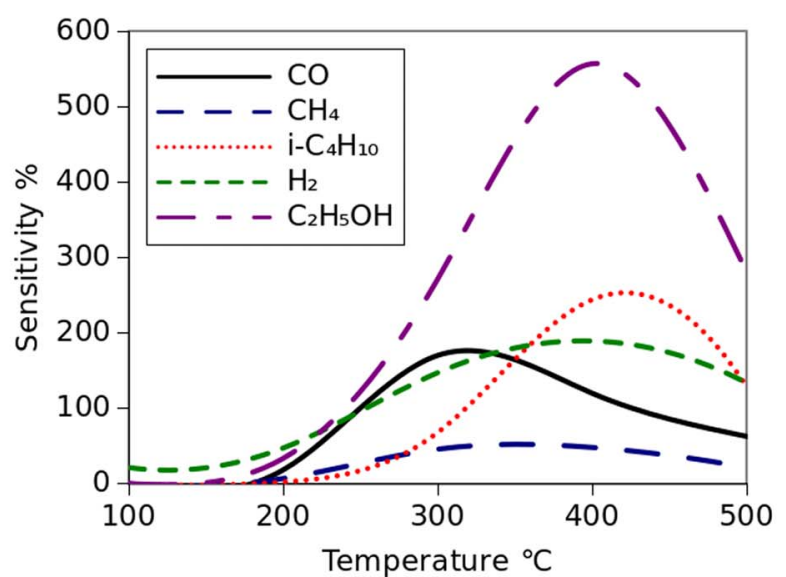

(b)

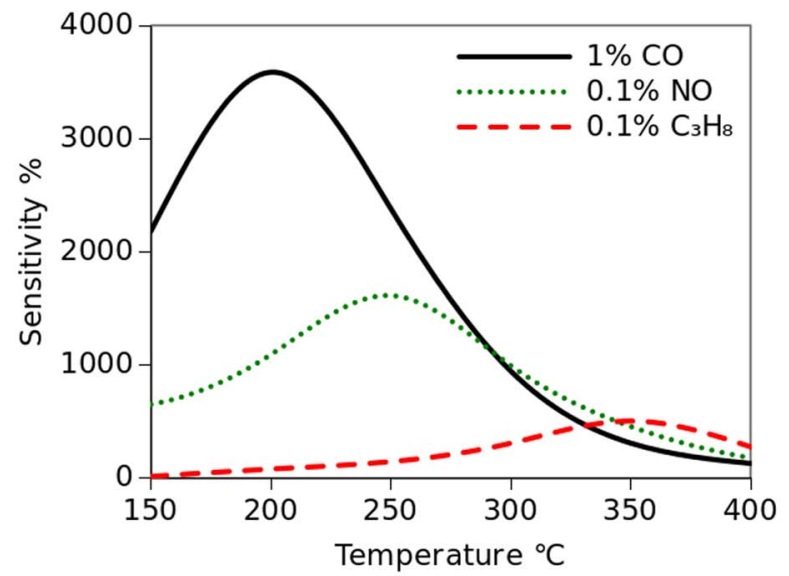

(d)

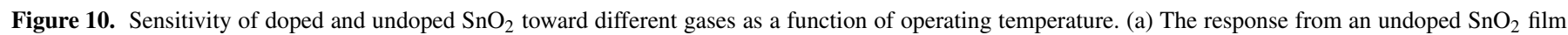

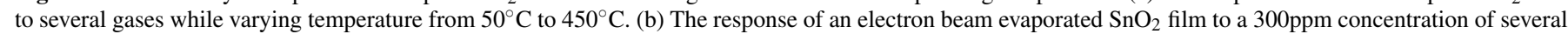

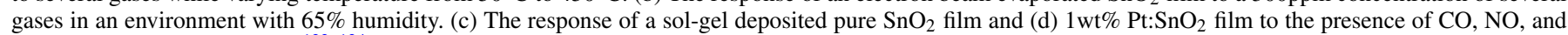
$\mathrm{C}_{3} \mathrm{H}_{8}$ at various temperatures. ${ }^{122-124}$ 


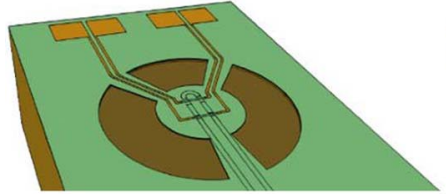

(a)

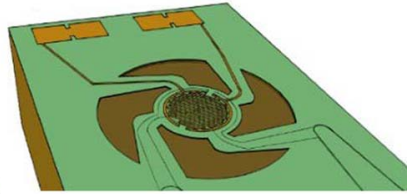

(b)
Figure 11. Structure of the SMO gas sensor membrane, used with the (a) microheater array and (b) dual-hotplate designs.

Dual-hotplate.-The dual-hotplate design is shown in Figure 9b and is a combination of a single circular microheater, suggested by Elmi et al., ${ }^{127}$ together with two passive micro-hotplates, suggested by Lahlalia et al. ${ }^{78}$ The hotplates are used for improved thermal uniformity in the active region. Even a small variation in the temperature over the sensing element can lead to baseline drift, changing the baseline resistivity of the SMO sensor, thereby requiring frequent calibrations. The fact that the additional hotplates are electrically passive ensures that the power consumption is kept at a minimum, which is also a critical factor to guarantee stability of the sensor baseline.

A new membrane shape has been designed to accompany the dualhotplate structure, formed by four curved micro-bridges, in order to provide improved insulation against heat losses to the substrate, shown in Figure 11b. In Figure 11a the three-armed membrane shape, used with the microheater array, is shown. The differences in the mechanical stability of the two membrane types, estimated by modeling, are discussed in the following section. Of note is the lack of sharp corners, but the use of rounding instead. This is in order to reduce the effects of current crowding, which could lead to void or hillock formation and eventual cracking.

\section{Modeling and Analysis of SMO Sensor Structures}

In order to have a complete picture of the fabrication, reliability, and operation of SMO gas sensors, measurements alone do not suffice. Many modeling techniques and simulation tools are essential in order to provide an in-depth analysis of the interplay between different materials in this complex structure. In this section the essential modeling approaches used to gain a deeper understanding of SMO gas sensors are described. These include models for the fabrication of the CMOS-integrable devices, electro-thermal modeling using finite element methods (FEM) and compact models, as well as mechanical simulations using FEM. With regard to the microheater operation, the focus is on its power dissipation, thermo-mechanical properties, and mechanical stability, ${ }^{128}$ while the $\mathrm{SnO}_{2}$ metal oxide is discussed in terms of its conductive response in the presence of a target gas in the environment ${ }^{87}$ in the next section.

SMO sensor fabrication techniques.-The complexity in the fabrication of SMO gas sensor devices is heightened due to the need for a thermally-isolated membrane. As mentioned earlier, this membrane can be generated by etching from the back of the wafer using deep reactive ion etching (DRIE) techniques or from the top, through openings in the membrane using wet chemical etching or plasma etching. When etching from the back, a closed membrane is generated, while etching through holes in the top results in a suspended membrane with suspension beams connecting the active region to the rest of the structure. ${ }^{66}$ The closed membrane thicknesses are in the range between $1 \mu \mathrm{m}$ and $2 \mu \mathrm{m}$, but the required back-side etching techniques make the process more expensive and not fully CMOS-compatible. ${ }^{97,129}$ For this reason, we concentrate our studies on the suspended-membrane sensor.

A thorough analysis has been performed on a suspended membrane sensor with a typical geometry and an active area of $100 \mu \mathrm{m} \times$ $100 \mu \mathrm{m}$ using both wet chemical potassium hydroxide $(\mathrm{KOH})$ and $\mathrm{SF}_{6}$ plasma models. ${ }^{72}$ The simulation for $\mathrm{KOH}$ etching was achieved using the model described in Ref. 130 and the ViennaTS tool. ${ }^{131}$ Using a 150 minute etch with a $\mathrm{KOH}$ concentration of $30 \%$ and a temperature of $70^{\circ} \mathrm{C}$ the suspended membrane was generated with a $100 \mu \mathrm{m}$ hole. The silicon etch rate is dependent on the crystallographic orientation and, under the noted etch conditions, the rates for directions $<100>$, $<110>,<111>$, and $<311>$ were found to be $R_{100}=13.3 \mathrm{~nm} / \mathrm{s}$, $\mathrm{R}_{110}=24.2 \mathrm{~nm} / \mathrm{s}, \mathrm{R}_{111}=0.1 \mathrm{~nm} / \mathrm{s}$, and $\mathrm{R}_{311}=23.9 \mathrm{~nm} / \mathrm{s}$, respectively. Although the $\mathrm{KOH}$ structure displays a very clean geometry without undesirable lateral etching (Figure 12), the process can be very corrosive to the surrounding devices. For this reason, further analyses of the plasma etching on the same geometry have been carried out. This process is less corrosive and more compatible with CMOS fabrication, but suffers from profound lateral etching, as shown in Figure 12.

Simulations of plasma etching have been carried out using the physics model described by the group at the University of California in Refs. 132 and 133, implemented within the ViennaTS tool. ${ }^{131}$ The key idea is to use a stochastic approach to the particles, which are present in the plasma chamber and which have an influence on the etching process. The particles can either be neutral, representing a chemical etch component, or ionic, representing a physical etch component. The chemical etch component is the primary contributor to the lateral etching underneath the eventual suspended membrane. However, since the lateral etching proceeds in all directions, the overall size required by the sensor increases when this method is applied. Using $\mathrm{SF}_{6}$ plasma chemistry with a fluorine flux of $1 \times 10^{19} \mathrm{~cm}^{-2} \mathrm{~s}^{-1}$ and disregarding any ion involvement, a 300 second etch was sufficient to expose the membrane to the level shown in Figure 12. The ion involvement was not simulated because it only adds to the vertical etching, while the goal of a membrane release etch is to ensure a maximum lateral etching underneath the membrane. This is achieved using a fully isotropic etch, meaning removing the vertical influence of the ions.

In follow-up analysis, it was found that the lateral etching had no adverse effects on the stress distribution in the active sensor region..$^{72,88}$ The modeled post-processing stress is a combination of the residual stresses in the layers which make up the entire membrane. The control of the residual stress in multilayered structures is crucial for its stability. The typical values for the as-deposited stress in the $\mathrm{Si}_{3} \mathrm{~N}_{4}$

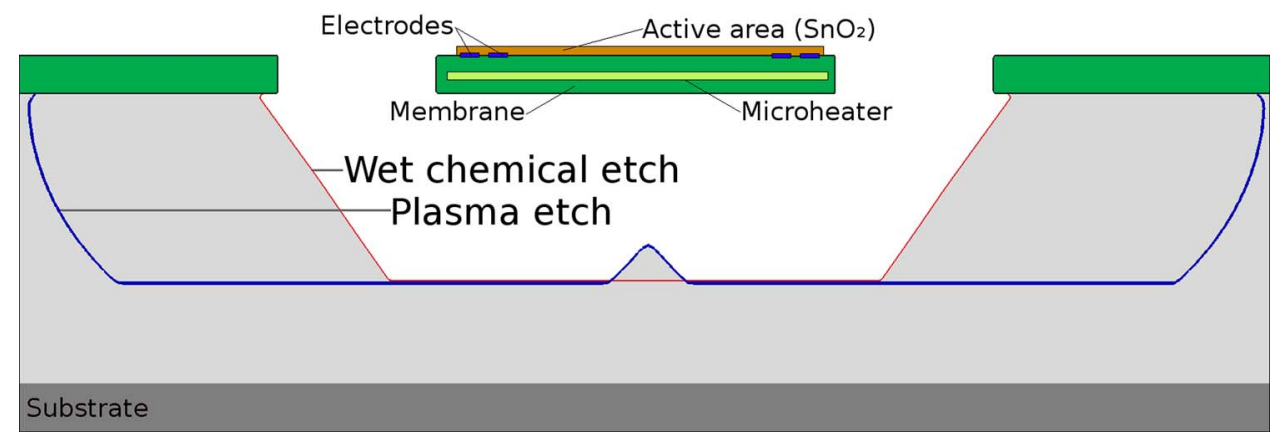

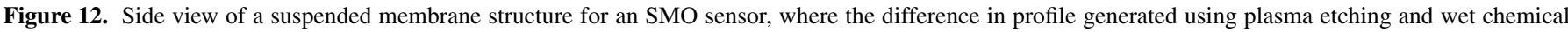
etching is pronounced. 
and $\mathrm{SiO}_{2}$ layers are approximately $1 \mathrm{GPa}$ and $-320 \mathrm{MPa}$, respectively. These values were applied in the simulations in Ref. 72 and then the structure was allowed to relax. The average resulting stress in both structures was found to be on the order of $300 \mathrm{MPa},{ }^{72}$ which is outside of the acceptable range of residual stress, which should remain below $100 \mathrm{MPa}$. The simulation nevertheless shows how the membrane deals with an applied external stress, which is most commonly the thermal stress during operation, since the membrane temperature regularly changes from several hundreds of Celsius to room temperature within short time spans. ${ }^{134}$

Another aspect of the sensor fabrication which has been investigated is the deposition of the sensing SMO film itself. This layer can be deposited in a variety of ways, including chemical vapor deposition, sputtering, pulsed-layer deposition, sol-gel process, rheotaxial growth and vacuum oxidation, and spray pyrolysis. ${ }^{72,88}$ Sputtering and spray pyrolysis are methods which are quite straight forward and cost effective to implement within the CMOS sequence. Using both etching methods, the resulting geometry was analyzed with a topography simulator ViennaTS ${ }^{131}$ in Ref. 135. Spray pyrolysis showed a more isotropic coverage around corners and edges; however, the spray pyrolysis deposition requires elevated temperatures $\left(400^{\circ} \mathrm{C}\right)$, meaning that a thermal stress can develop in the film due to the subsequent cooling to room temperature. This is not necessarily a disadvantage, since it results in having a film which is relatively stress-free at elevated temperatures, considering that the SMO film must be heated to temperatures in the range of $250^{\circ} \mathrm{C}$ to $500^{\circ} \mathrm{C}$ in order to activate sensing. ${ }^{88}$ The two main post-processing stress components analyzed are the intrinsic stress, which forms during the film growth, and the thermo-mechanical stress, which results due to the difference in the deposition temperature and the subsequent cooling to room temperature. ${ }^{88,136}$ The thermo-mechanical stress is a concern when elevated temperatures are used for the deposition process due to the differences in the CTE between the depositing material and the substrate.

Electro-thermal analysis.-Understanding the electro-thermal behavior of the sensor is essential to understanding its long-term reliability, sensitivity, and selectivity. In a recent work ${ }^{97}$ several means of characterizing the power-temperature behavior of a TaAl microheater was examined. These included the use of resistance temperature detectors (RTDs) of platinum and chromium silicon ( $\mathrm{CrSi}$ ) and comparing the characterizations to finite element simulations and an analytical model. Modeling the electro-thermal behavior of the sensor and microheater in particular is essential to understanding the heat losses, shown in Figure 13, and thereby to minimizing the power dissipation in the designed sensors. The analysis is usually performed in a finite element environment, which requires meshing the full geometry

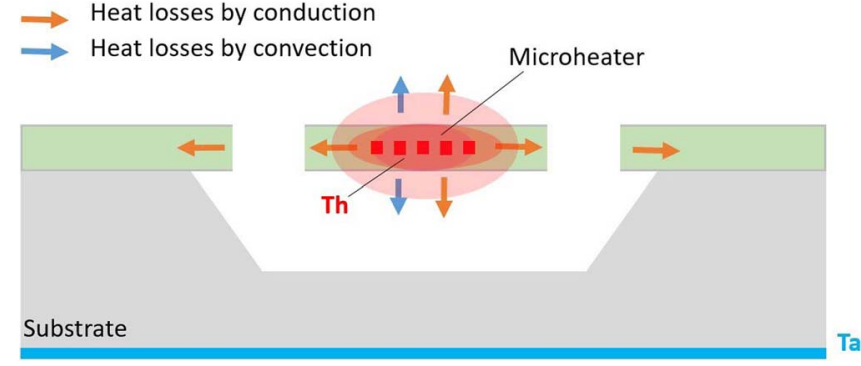

Figure 13. Heat loss mechanisms in the SMO gas sensor and surroundings, where $\mathrm{Th}$ is the microheater temperature, required for sensor operation and $\mathrm{Ta}$ is the ambient temperature.

followed by memory and computationally intensive simulations. The ambient conditions in the model are set to room temperature $\left(20^{\circ} \mathrm{C}\right)$ and the heat transfer coefficient with the air is set to a value derived from a temperature-dependent function based on the fluid motion and conduction through air. ${ }^{97}$ The value is calculated using a Nusselt number, assuming laminar flow. ${ }^{137}$

The main sources of heat loss, depicted in Figure 13 are conduction and convection through the air and conduction through the membrane. There is a slight loss to radiation in the air, but this is relatively minor when compared to other losses. In Ref. 134 the heat conduction for a typical $100 \mu \mathrm{m} \times 100 \mu \mathrm{m}$ sensor structure was calculated, which required a total of $32.5 \mathrm{~mW}$ to heat to $400^{\circ} \mathrm{C}$. Of the total dissipated $32.5 \mathrm{~mW}, 18.9 \mathrm{~mW}$ was lost to the air conduction above the membrane and $1.3 \mathrm{~mW}$ to the air conduction below the membrane. A total of $12.2 \mathrm{~mW}$ was lost to the conduction through the membrane beams, while a minimal $0.16 \mathrm{~mW}$ was lost to radiation. Therefore, we can safely conclude that the bulk of the heat lost in a suspended membrane is through the membrane and air conduction through the top exposure to the air. For a closed membrane, most of the heat loss will be conduction through the solid material, along the sides of the active area and through the silicon wafer.

With this in mind, the microheater array and dual-hotplate designs, presented in the previous section, were optimized and significantly improved, resulting in a total power dissipation of only $9.31 \mathrm{~mW}$ and $8 \mathrm{~mW}$, respectively, when operating at $350^{\circ} \mathrm{C}$. In Figure 14, the thermal distribution for the two designs is shown. Here, we also note that the temperature uniformity across the active region is very good, with variations below $10^{\circ} \mathrm{C}$ or below $3 \%$. In the microheater array design in Figure 14b we also see two active regions with two different target temperatures operating simultaneously. This design allows us to heat the sensor to $270^{\circ} \mathrm{C}$ and $350^{\circ} \mathrm{C}$ at the same time, meaning that we can

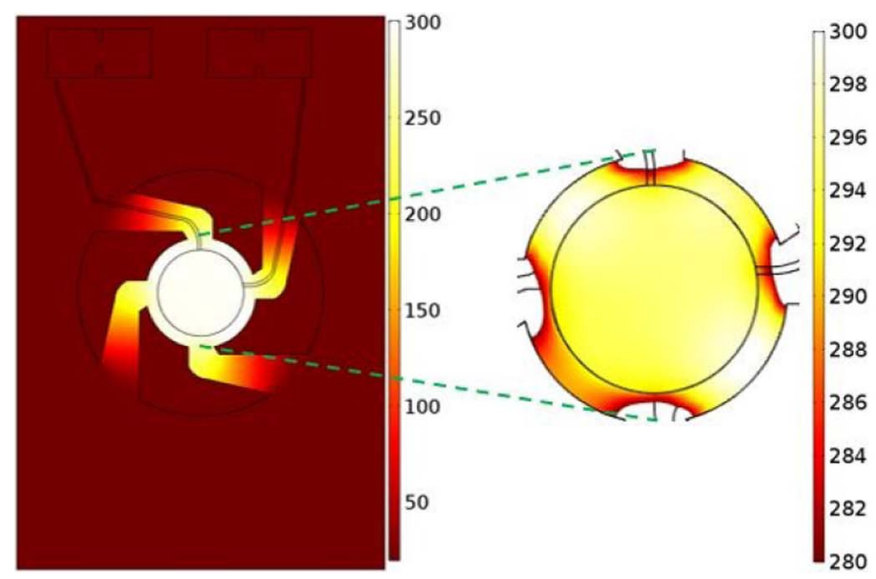

(a)

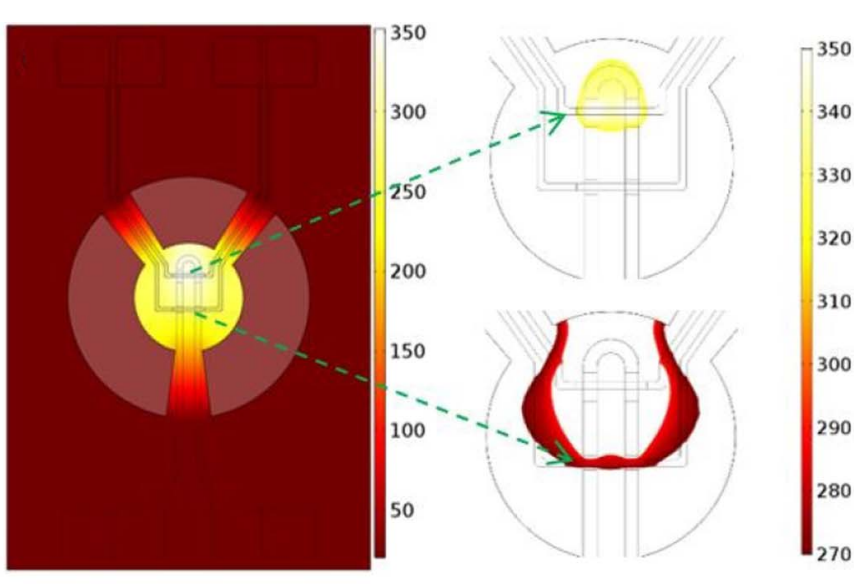

(b)

Figure 14. Simulation of the thermal distribution $\left({ }^{\circ} \mathrm{C}\right)$ in a (a) dual-hotplate design and (b) a microheater array design. The microheater array allows for concurrent operation at multiple temperatures. 


\begin{tabular}{|c|c|}
\hline Thermal parameter & Electrical equivalent \\
\hline Temperature (K) & Voltage (V) \\
\hline Specific heat $(\mathrm{J} / \mathrm{Kg} \cdot \mathrm{K})$ & Permittivity $(\mathrm{F} / \mathrm{m})$ \\
\hline Thermal resistivity $(\mathrm{K} \cdot \mathrm{m} / \mathrm{W})$ & Electric resistivity $(\Omega \cdot \mathrm{m})$ \\
\hline Thermal resistance $(\mathrm{K} / \mathrm{W})$ & Electric resistance $(\Omega)$ \\
\hline Heat flow (W) & Current (A) \\
\hline Thermal conductivity $(\mathrm{W} / \mathrm{K} \cdot \mathrm{m})$ & Electric conductivity $(\mathrm{S} / \mathrm{m})$ \\
\hline Heat (W.s) & Charge $(\mathrm{A} \cdot \mathrm{s})$ \\
\hline Thermal capacitance $(\mathrm{J} / \mathrm{K})$ & Electric capacitance $(\mathrm{F})$ \\
\hline
\end{tabular}

selectively test for several gases (Figure 10) during a single current pulse.

In order to eliminate the need for FEM simulations and the associated meshing and computational requirements, a model has been developed, which can be used to represent the temperature field and calculate the electro-thermal behavior of the sensor. ${ }^{78}$ Although FEM is a very powerful tool and can accurately predict many electro-thermalmechanical phenomena, it requires a mesh to represent the entire sensor structure as well as the surrounding air. When dealing with very thin layers in a large structure, the aspect ratios required are large and the number of mesh elements can quickly increase to levels not easily manageable on a standard desktop computer. In addition, methods such as FEM or finite volumes, generate the complete set of equations at each explicit node and for every temperature, meaning a large number of equations must be solved. As an alternative, an analytical model is frequently used, which emulates an integrated circuit (IC) using thermal elements, represented by their electrical equivalents. The entire structure is effectively broken down into small segments, each represented with a parallel thermal resistor-capacitor-inductor circuit. In Table III the relationship between the heater's thermal properties and an electrical component equivalent is shown. Using this strategy, the complete sensor geometry is discretized and an IC, which very accurately calculates the heat loss by convection, is generated. The power of this method is made obvious in Ref. 97, where the results are compared with measurements and an FEM simulation, shown in Figure 16. The method was able to accurately calculate the powertemperature relationship even for complex microheater geometries.

Mechanical reliability analysis.-One of the major concerns regarding the lifetime of SMO gas sensors is the long-term stability of the suspended membrane. The high temperatures, combined with a variety of materials with different CTEs, can lead to the buildup of excessive stress in the membrane and eventual cracking or delamination failures. ${ }^{88}$ The stress in the membrane is a combination of the residual stresses in the layers which make up the complete membrane stack. This stress builds up due to two factors: intrinsic stress during deposition and thermal stress due to the post-deposition cooling to room temperature. ${ }^{41,88}$ The deposition of metal and semiconductor layers most often follows the Volmer-Weber growth mode, which involves the generation of islands, which then grow and impinge on each other, forming grains and grain boundaries. ${ }^{136}$ This type of stress buildup has been studied in the membrane layers as well as in the SMO film itself, ${ }^{138}$ showing that spray pyrolysis deposited films suffer from a higher stress at room temperature than sputtered films. However, during operation at elevated temperatures, the sputtered films experience increased thermal stresses.

Using FEM, the effects of the as-deposited stress on the deformation in the membrane layer have been studied. Surprisingly, it has been found that the thermal stress distribution in the active area induced during thermal cycling is unaffected by the method of the membrane formation, whether using $\mathrm{KOH}$ or plasma etching. ${ }^{72}$ However, the maximum displacement, at the center of the membrane (with a $100 \mu \mathrm{m} \times 100 \mu \mathrm{m}$ active region), was found to be $8 \mu \mathrm{m}$ for the plasmaetched membrane, compared to $5 \mu \mathrm{m}$ for the $\mathrm{KOH}$-etched membrane.

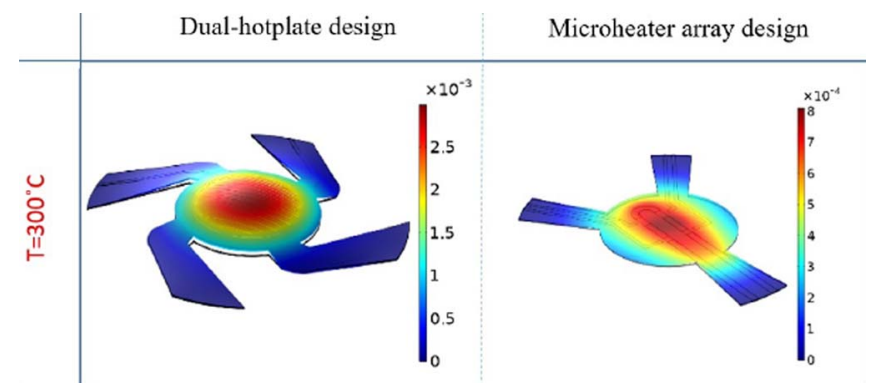

Figure 15. Total displacement $(\mathrm{mm})$ of the membrane stack when heated from room temperature $\left(20^{\circ} \mathrm{C}\right)$ to $300^{\circ} \mathrm{C}$.

This is due to the effective expansion in the membrane width due to the lateral etching component during plasma etching. The displacement in the novel membrane structures for the microheater array and dual-hotplate designs from Ref. 78, described earlier, are shown in Figure 15. There, the displacement is induced due to the thermal stress generated after heating the structure from $20^{\circ} \mathrm{C}$ to $300^{\circ} \mathrm{C}$. We note a displacement of about $3 \mu \mathrm{m}$ for the 4-beam design and about $0.8 \mu \mathrm{m}$ for the 3-beam design. This means that the designs both improve upon the generic membrane structure from Figure 12 and that the 3-beam membrane displaces much less, indicating that it has a reduced likelihood of crack formation on the surface of the sensing element.

\section{Sensing Mechanism of the SMO Film}

A complete understanding of the sensing mechanism of the SMO sensor is not yet available, but significant progress has been made recently in understanding conductivity and surface charge effects in one of the most frequently used $\mathrm{SMOs}, \mathrm{SnO}_{2}$. The change in resistance of the sensing layer, when exposed to a target gas, is the factor which determines its sensitivity. Because the resistivity changes in the presence of a reducing or oxidizing gas, understanding the change in the resistivity, or conductivity, is the key to understanding the SMO's sensing mechanism. As is the case for chemical sensors in general, the sensing effect is based on reception and transduction. The reception is that of an analyte gas with an SMO layer, through a surface chemical reaction, and transduction is of the changes at the SMO layer surface,

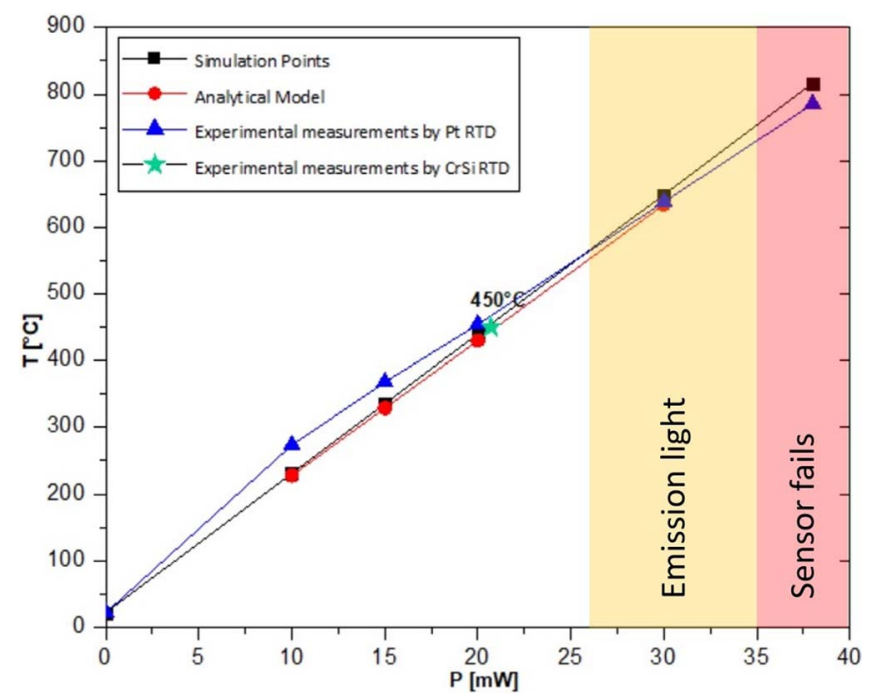

Figure 16. Temperature as a function of applied power to the microheater in a design presented in Ref. 97. The figure shows the power of the analytical model to replicate the realistic electro-thermal behavior of a SMO sensor. ${ }^{97}$ 
which influence the electronic conductive properties of the sensing film and charge transport therein. ${ }^{139}$

SMO conductivity. $-\mathrm{SnO}_{2}$ is a wide bandgap n-type semiconductor, which means its donor states are related to oxygen vacancies and that electrons are its majority carriers during conduction. The calculated bandgap between the valence and conduction bands is $3.6 \mathrm{eV},{ }^{140}$ which was also measured and confirmed experimentally. ${ }^{141}$

Drift-diffusion.-The electrical conduction of SMO films is modeled using drift-diffusion equations. ${ }^{142}$ The drift diffusion equations are commonly used when modeling conduction in a semiconductor and are defined by the diffusion and drift components, controlled by the number of charge carriers, which can be electrons or holes, the mobility of the charge carriers, and the applied electric field. The drift diffusion equations are summarized by

$$
\begin{gathered}
\nabla \cdot \overrightarrow{j_{n}}=R, \\
\nabla \cdot \overrightarrow{j_{p}}=-R, \\
\overrightarrow{j_{n}}=q n \mu_{n} \vec{E}+q D_{n} \nabla \mathrm{n}, \\
\overrightarrow{j_{n}}=q p \mu_{p} \vec{E}-q D_{p} \nabla \mathrm{p},
\end{gathered}
$$

where $\overrightarrow{j_{n}}$ and $\overrightarrow{j_{p}}$ are the current densities for n-type and p-type semiconductors, respectively; $R$ is the recombination rate; $\mu_{n}$ and $\mu_{p}$ are the mobilities of electrons and holes, respectively; $D_{n}$ and $D_{p}$ are the diffusion constants for electrons and holes, respectively; $q$ is the elementary charge of an electron, and $\vec{E}$ is the applied electric field. The $n$ and $p$ values are the electrons and hole concentrations in an n-type and p-type semiconductor, respectively. When a potential $V$ is applied across the film, the electric field $\vec{E}$ is derived from the solution to the Poisson equation, with appropriate boundary conditions, which relate the derivative of the electrical potential to the surface charge density $\rho$ and the permittivity $\epsilon_{0} \epsilon_{r}$ of the material with

$$
-\nabla \cdot\left(\epsilon_{0} \epsilon_{r} \nabla \mathrm{V}(\overrightarrow{\mathrm{x}})\right)=\rho .
$$

The boundary conditions used are Dirichlet at the ohmic contacts, where a particular value for the potential is set, and Neumann conditions elsewhere. The electric field is calculated by the negative value of the applied potential gradient:

$$
\vec{E}(\overrightarrow{\mathrm{x}})=-\nabla \mathrm{V}(\overrightarrow{\mathrm{x}})
$$

In an n-type film, such as is the case with $\mathrm{SnO}_{2}$, only Equations 2 and 4 are considered using electrons as majority carriers, while in a p-type film, such as $\mathrm{CuO}$, the majority carriers are holes and they would have to be considered by solving 3 and 5. The discussion in the remainder of this sections deals with n-type materials only and more specifically with $\mathrm{SnO}_{2}$. How the equation would change when p-type materials are used can be extrapolated from this discussion.

Furthermore, it is generally assumed that the depletion layer thickness is in comparable dimension to the mean free path of the charge carriers, allowing to eliminate the diffusion component from 4 and 5 , thereby removing $q D_{n} \nabla \mathrm{n}$ and greatly simplifying the problem. Therefore, from 4 we extract the conductivity $\sigma=\overrightarrow{j_{n}} / \vec{E}$, which can now be defined as a combination of both the concentration $n$ and the mobility $\mu_{n}$ with

$$
\sigma=q \cdot n \cdot \mu_{n} .
$$

The electron concentration $n$ and the electron mobility $\mu_{n}$ can vary significantly with temperature, even in a fully inert environment; ${ }^{143}$ therefore, also the conductivity is significantly influenced by temperature. The influence of temperature on the electron mobility and concentration is depicted in Figure 17, where the resulting temperaturedependent conductivity is also shown. The conductive behavior of the

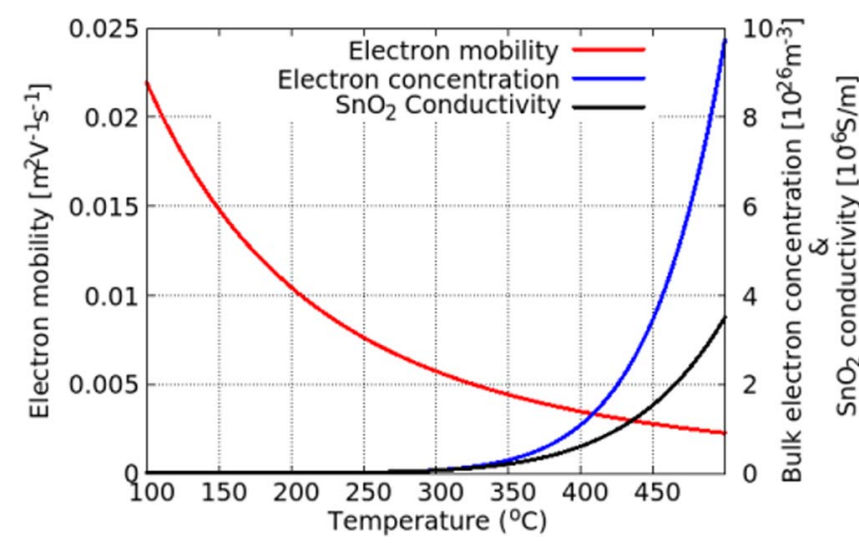

Figure 17. Temperature dependence on the electron concentration (n) and mobility $\left(\mu_{\mathrm{n}}\right)$ of $\mathrm{SnO}_{2}$. The conductivity is a combination of both $\mathrm{n}$ and $\mu_{\mathrm{n}}$ by $\sigma=q \cdot n \cdot \mu_{n}$.

SMO sensor is not unlike a transistor; however, instead of directly applying a potential at the gate, a potential is applied indirectly through the accumulation of charges at the surface. This charge stems from the ionosorption of gases at the SMO surface. Which sections of the surface are able to adsorb charge depends on the type of sensing material. A porous SMO film is most commonly used, due to its granular structure, which increases its effective surface to volume ratio. A high surface to volume ratio exacerbates surface effects in the film and is therefore desired for sensing devices.

Porous SMO films.-The typical SMO gas sensors in use today consist of a porous film deposited on an insulating substrate. The porous film is comprised of many grains, all of which have their own effective external surface, which can be exposed to a target gas, thereby increasing the surface to volume ratio. The conductivity involves conduction through grain-grain, grain-bulk, and grain-electrode interfaces, as described in some detail in Ref. 144. The limiting conducting factor is the grain-grain interface, since this has the highest resistance due to the limited charge carrier concentration and reduced mobility at the surfaces of the grains. Since the mobility of the charge carriers remains unchanged during gas molecule adsorption, the resistance depends purely on the charge concentration. ${ }^{139}$ The charge concentration can be manipulated through the sharing of charges with an adsorbed molecule, which simplistically describes the sensing mechanism.

Modeling the conductivity at the grain-grain interface assumes that the Schottky approximation is valid, meaning that all donors are fully ionized. In this case the electron concentration at the surface is described by the Boltzmann distribution

$$
n_{s}=N_{D} \exp \left(-\frac{q V_{s}}{k_{B} T}\right),
$$

where $N_{D}$ is the donor density, $q V_{s}$ is the energy of the surface band bending, which is depicted in Figure $19, k_{B}$ is the Boltzmann constant, and $T$ is the temperature. The resistance of the sensing layer is thereby proportional to the surface band bending $q V_{s}$. When modeling the sensor, the surface charges can be used to calculate a surface potential $V_{s}$, which is solved for using the Schottky relation

$$
V_{s}=-\frac{q N_{e f f}^{2}}{2 \epsilon_{0} \epsilon_{r} N_{D}},
$$

where $N_{\text {eff }}$ is the sum of the electrons which gain enough energy to reach the surface together with the external electrons donated from adsorbed or ionosorped gas ions. ${ }^{145}$ The resistance of the sensing layer is therefore proportional to the surface band bending according to

$$
R \propto \exp \left(-\frac{q V_{s}}{k_{B} T}\right) .
$$




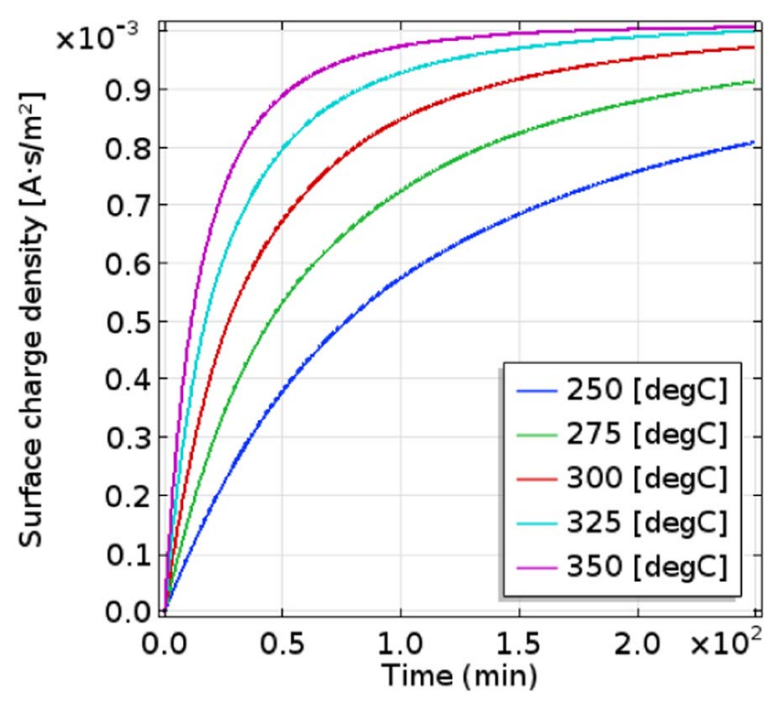

Figure 18. Surface charge density $\left[\mathrm{A} \cdot \mathrm{s} / \mathrm{m}^{2}\right]$ in a synthetic air environment, with $80 \% \mathrm{~N}_{2}$ and $20 \% \mathrm{O}_{2}$. This shows the effects of the surface ionosorption with $\mathrm{O}^{-}$and $\mathrm{O}^{2-}$ ions.

Since the sensor signal describes the change in resistance, the sensitivity can also be described by a difference in the surface band bending.

In summary, the ionosorption of gas molecules attracts electrons from the $\mathrm{SnO}_{2}$ bulk, resulting in band-bending at the interface between the material and the surrounding gas. The amount of band bending is proportional to the effective concentration of localized surface electrons $N_{\text {eff }} .{ }^{142}$ In Figure 18, the surface charge density is plotted versus time at various temperatures in a synthetic air environment, where $\mathrm{O}^{-}$ and $\mathrm{O}^{2-}$ ions are adsorbed at the surface. It is evident that the surface charge density saturates in accordance with the total number of ionosorption sites available. Also of note is the influence of temperature; increasing the temperature from $250^{\circ} \mathrm{C}$ to $350^{\circ} \mathrm{C}$ allows for the oxygen ions to reach saturation more quickly.

Surface chemisorption.-The sensing of a desired gas, such as $\mathrm{CO}$, occurs after a $\mathrm{CO}$ molecules reacts with a previously ionosorped oxygen atom, releasing it from the surface, as depicted in Figure 19. The chemical reactions taking place at the surface during the oxygen adsorption mechanism has been researched extensively. Molecular species and atomic species have been proposed to interact with the $\mathrm{SnO}_{2}$ surface. ${ }^{144,146}$ Degler ${ }^{139,147}$ recently studied the active oxygen

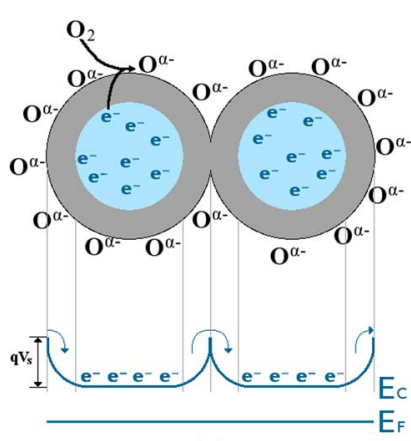

(a)

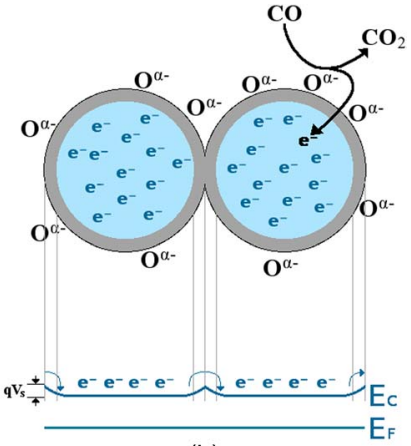

(b)
Figure 19. Gas sensing and conduction mechanism for a porous metal oxide, where the oxygen and reducing gas can penetrate to interact with each grain. In (a) the oxygen ion is adsorbed on the grain surface, forming a depletion region around the grain. In (b) after the introduction of a reacting gas such as $\mathrm{CO}$, which reacts with some of the oxygen ions, the depletion region thickness is reduced.

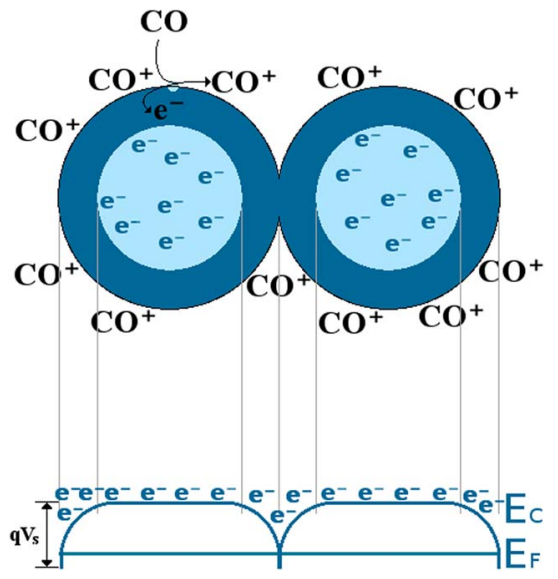

Figure 20. It is theorized that, after depleting all the oxygen at the SMO surface, $\mathrm{CO}$ gas molecules can directly interact with surface atoms, thereby donating atoms to the grain and forming an accumulation layer. ${ }^{40}$

species in $\mathrm{SnO}_{2}$ sensors using IR spectroscopy and summarized the steps in the transformation of an atmospheric oxygen $\left(O_{2, g a s}\right)$ to a lattice oxygen $\left(O_{O}\right)$ as

$$
\frac{1}{2} O_{2, \text { gas }}+V_{O}^{2+}+2 e^{-} \rightleftharpoons O_{O}
$$

by including all intermediate steps and species, in the following set of reactions:

$$
\begin{gathered}
O_{2, g a s} \rightleftharpoons O_{2, a d s} \\
O_{2, a d s}+e^{-} \rightleftharpoons O_{2, a d s}^{-} \\
O_{2, a d s}^{-}+e^{-} \rightleftharpoons O_{2, a d s}^{2-} \\
O_{2, a d s}^{2-} \rightleftharpoons 2 O_{a d s}^{-} \\
O_{a d s}^{-}+e^{-} \rightleftharpoons O_{a d s}^{2-} \rightleftharpoons O_{O}
\end{gathered}
$$

The adsorption of carbon monoxide $(\mathrm{CO})$ molecules then proceeds according to:

$$
\mathrm{CO}_{\text {gas }}+\mathrm{O}_{2, a d s}^{2-}+(2-\alpha) \cdot e^{-} \rightleftharpoons\left(\mathrm{CO}_{3}\right)^{\alpha-}
$$

$$
\mathrm{CO}_{\text {gas }}+\mathrm{O}_{O} \rightleftharpoons\left(\mathrm{CO}_{2}\right)_{O}
$$

Based on the above description, sensing reducing gases, such as $\mathrm{CO}$, would only be detectible in the presence of oxygen; however, it was recently found that, even without $\mathrm{O}_{2}$, the $\mathrm{SMO}$ film continues to show a sensing response, while no oxidation product $\left(\mathrm{CO}_{2}\right)$ was found in the exhaust. ${ }^{148}$ This led to the expansion of the above description to include a subsequent step, which is the formation of a donor species (e.g. $\mathrm{CO}^{+}$) which adsorbs and directly injects electrons into the conduction band. ${ }^{148}$ This is shown graphically in Figure 20, where the injection of electrons leads to the formation of a narrow accumulation layer. An explanation for this behavior is the adsorption of $\mathrm{CO}$ on a tin $(\mathrm{Sn})$ site, whereby charge is transferred directly from the $\mathrm{CO}$ molecule to the solid: ${ }^{149,150}$

$$
C O_{\text {gas }} \rightleftharpoons C O_{a d s}
$$

In summary, there are several cases of gas reception on $\mathrm{SnO}_{2}$ films and they each behave somewhat differently, but nevertheless are based on the interplay of the surface reduction and re-oxidation by atmospheric oxygen. The following situations are suggested to describe all possible 


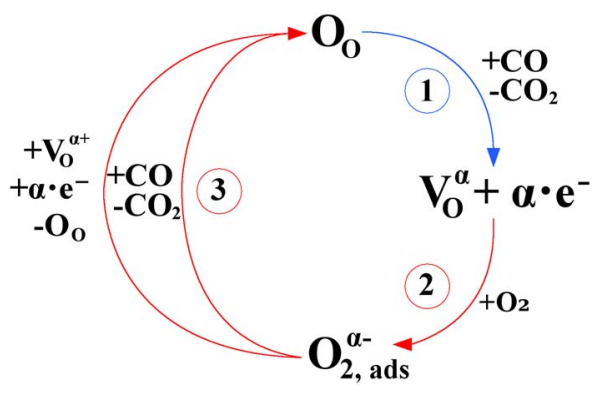

Figure 21. Proposed surface chemistry model for the reception of small reducing gases on a pristine $\mathrm{SnO}_{2}$ surface. ${ }^{147}$

reactions at elevated temperatures for simple molecules, which do not produce species through intermediate surface reactions: ${ }^{139,151}$

1. With no reacting gas present (e.g. $\mathrm{N}_{2}$ ambient) the surface energy bands are flat and the number of charges on the surface corresponds to the number of charges in the bulk.

2. In the presence of oxygen gas, the SMO film's surface oxygen vacancies are filled and a charge is trapped at the formed lattice oxygen species, thereby ensuring that the donor concentration at the surface is lower than that in the bulk and creating a depletion layer.

3. If only a reacting gas $(\mathrm{CO})$ is present, with no atmospheric oxygen, a surface oxygen vacancy reacts with $\mathrm{CO}$, thereby reducing the surface. Therefore, the donor concentration at the surface is increased, when compared to the bulk, and a thin accumulation layer is formed, without the release of oxidation by-products.

4. When both $\mathrm{O}_{2}$ and $\mathrm{CO}$ are found in the atmosphere, the surface oxygen will be formed by adsorption to the oxygen vacancy site. This will subsequently be removed during oxidation of $\mathrm{CO}$ to form $\mathrm{CO}_{2}$ and the surface will be continuously re-oxidized. In high concentrations of atmospheric oxygen, this will lead to a reduction in the depletion layer. In low oxygen concentration, or in the presence of interfering gases (e.g. $\mathrm{H}_{2} \mathrm{O}$ ), the surface oxygen vacancy concentration is higher than that in bulk and an accumulation layer is formed.

While the adsorption of CO through the intermediate adsorption of oxygen is discussed here, several studies also deal with gas detection through the intermediate adsorption of water $\left(\mathrm{H}_{2} \mathrm{O}\right)$ instead. For an in-depth analysis of the use of hydroxide as an intermediate species, further reading in Refs. 139,151 is recommended.

Surface reaction model for $\mathrm{CO}$ on $\mathrm{SnO}_{2}$.- The reactions described above are the closest researchers have come to understanding the surface interactions leading to gas detection in SMO films. This model is quite intricate and involves many steps with many reaction constants, as summarized in Figure 21. ${ }^{147}$ With this model, the full simulation of a complex structure is very difficult and cumbersome, with several fitting parameters necessary, including the adsorption and desorption rates of all reactions. When attempting to simulate a sensor, whether it be a porous film ${ }^{40}$ or a nanowire, ${ }^{142}$ several estimations are generally made. Primarily, the intermediate reactions are left out and the adsorption of oxygen, water vapor, and subsequently carbon monoxide is treated using three reactions ${ }^{40}$

$$
\begin{gathered}
\frac{1}{2} O_{2, \text { gas }}+S+\alpha \cdot e^{-} \rightleftharpoons O_{S}^{-\alpha} \\
H_{2} O_{g a s}+2 \cdot S n_{S n}+O_{S}^{-\alpha} \rightleftharpoons 2 \cdot(\operatorname{SnOH})+\alpha \cdot e^{-}+S \\
C O_{\text {gas }}+O_{S}^{-\alpha} \rightarrow C O_{2, \text { gas }}+\alpha \cdot e^{-}+S,
\end{gathered}
$$

where $S$ is a surface adsorption site, $\alpha=1$ for strongly ionized oxygen, $\alpha=2$ for doubly ionized oxygen, $O_{S}^{-\alpha}$ is a chemisorbed oxygen species, and $e^{-}$represents an electron in the conduction band. It becomes evident that only the surface oxidation/re-oxidation (situation 4. above) is treated in this model, as a comprehensive model for the situations 2 . and 3 . are still a work in progress. The mass action law stemming from the reactions described by 21,22 , and 23 can then be applied to generate a rate equation for the oxygen surface coverage $\left[O_{S}^{-\alpha}\right]$ in the steady state

$$
\begin{gathered}
\frac{d\left[O_{S}^{-\alpha}\right]}{d t}=k_{a d s} \cdot p_{o_{2}}^{\frac{1}{2}} \cdot[S] \cdot n_{S}^{\alpha}-k_{d e s} \cdot\left[O_{S}^{-\alpha}\right] \\
-k_{H_{2} O, a d s} \cdot p_{H_{2} O} \cdot\left[S n_{S n}\right]^{2} \cdot\left[O_{S}^{-\alpha}\right] \\
+k_{H_{2} O, d e s} \cdot[O H]^{2} \cdot e \cdot n_{S}^{\alpha}-k_{\text {react }} \cdot\left[O_{S}^{-\alpha}\right] \cdot p_{C O},
\end{gathered}
$$

where the square brackets [ ] denote surface coverage, $k_{\text {ads } / \text { des }}$ are the reaction constants for oxygen adsorption/desorption, $k_{\mathrm{H}_{2} O \text { Oads/des }}$ are the reaction constants for water vapor adsorption/desorption, $k_{\text {react }}$ is the reaction constant for Equation 23, $p_{\mathrm{O}_{2} / \mathrm{CO}}$ are the partial pressures of $\mathrm{O}_{2} / \mathrm{CO}$, and $n_{S}$ is the concentration of electrons at the surface. Since the concentration of tin atoms at the surface is very large compared to those of the hydroxyl groups and with surface coverage $\theta=\left[O_{S}^{-\alpha}\right] /[S]$, the sensor signal $S=R_{C O} / R_{\text {air }}$ can be modeled with a power law approximation

$$
S=\left(1+\frac{\left(k_{\text {react }} / k_{\text {des }}\right) \cdot p_{C O}}{\kappa \cdot p_{\mathrm{H}_{2} O}}\right)^{1 /(\alpha+\gamma)},
$$

where $\kappa=1+k_{H_{2} O, a d s} \cdot\left[S n_{S n}\right]^{2} / k_{\text {des }}$ and $\gamma$ is used to account for the effects of humidity. A deeper investigation for the modeling of power law exponent $n=1 /(\alpha+\gamma)$ has been performed by Hua et al. ${ }^{152-154}$ and is described in the next section.

However, after the oxygen is depleted, there is an observed switch in conduction mechanisms from depletion-layer controlled to accumulation-layer controlled, discussed in the previous section and illustrated in Figure 19 and Figure 20. This change is significant for transduction, since conductance is no longer proportional to the surface concentration of free charge carriers, but rather the average over the accumulation layer. The reception of gas molecules (e.g. CO) is also different, since they no longer only react with the adsorbed oxygen, but also with $\mathrm{SnO}_{2}{ }^{40}$ A fully worked out model for this interaction is not yet available, but the following surface reactions are suggested in Ref. 40:

$$
C O_{\text {gas }}+S_{D, C O} \rightleftharpoons C O_{D}^{+}+e^{-},
$$

$$
\mathrm{H}_{2} \mathrm{O}_{\text {gas }}+\mathrm{S}_{\mathrm{D}, \mathrm{H}_{2} \mathrm{O}} \rightleftharpoons \mathrm{H}_{2} \mathrm{O}_{D}^{+}+e^{-},
$$

The reaction suggests the direct adsorption of reactive species on the surface and the addition of an electron, which contributes to the formation of an accumulation layer. Experimentally it was shown that an approximate amount of downward band bending, $q V_{S}$ from Figure 20, results in a change in the film's resistance by ${ }^{40}$

$$
R \propto \exp \left(-\frac{q V_{s}}{2 k_{B} T}\right),
$$

which has an extra $\frac{1}{2}$ in the exponent, compared to 11 . Since it is expected that the donor species (e.g. $C O_{D}^{+}$) determines the sensing effect, the electron concentration will be proportional to the concentration of the adsorbing gas molecule (e.g. CO). The conductance is proportional to the average electron concentration in the accumulation layer, which is proportional to the square root of the electron surface concentration. This square root dependence of the conductance on the partial pressure of CO can explain the extra $\frac{1}{2}$ in the exponent in 28 . This phenomenon has yet to be fully described and modelled, with discussions still ongoing on what exactly causes the power law response experimentally observed in SMO sensors and how to properly 
model the response to the sensing, as given by $n=1 /(\alpha+\gamma)$ in 25 , which is discussed in the next section.

Power-law response.-Recently, Hua et al. ${ }^{152-154}$ investigated means to physically model the power-law response of SMO gas sensors. The exponent in the power-law response is one of the primary problems in fully understanding the sensing mechanism of MOS gas sensors and in providing an accurate physical model. The exponent was shown to be specific to the particular gas and temperature. ${ }^{152-154}$ However, other results also show the exponent varying with preparation conditions of the sensing film and the relative humidity. ${ }^{155}$

The model proposed by Hua et al. is summarized here, but for a more detailed discussion, the reader should refer to. ${ }^{152-154}$ The study has been performed by observing the power response of $\mathrm{SnO}_{2}$ to exposure to $\mathrm{CO}$ in the presence of dry air. The transducer model is built on two conducing mechanisms, the Schottky barrier model and grain model, while the receptor functions were calculated using the law of mass action for oxygen with different concentrations of reducing gas. The primary novelty in the work is the suggestion that the exponent $n$ can be fully broken down into two components, one dealing with the transducer function and one the receptor function. The receptor function $\mathrm{R}_{\mathrm{ce}}$ deals with the adsorption of oxygen and subsequent reaction with $\mathrm{CO}$ or $\mathrm{H}_{2}$, while the transducer function $\mathrm{T}_{\mathrm{s}}$ deals with the influence of the added charges on band bending and resistance:

$$
\frac{d R}{d P}=\frac{d R}{d N_{t}} \times \frac{d N_{t}}{d P}
$$

The only relevant transducer function, treated by the authors, is the double-Schottky barrier model across neighboring grains, visualized in Figure 19. $N_{t}$ is defined as the number of donors of the charge density $N_{D}$, found in the depletion region. Solving 9,10 , and 11 and substituting in the transducer function from 29 the authors determine the transducer function as

$$
\frac{d R}{d N_{t}}=\frac{N_{t}}{\left(L_{D} N_{D}\right)^{2}}=\frac{m}{L_{D} N_{D}},
$$

where $L_{D}$ is the Debye length and $m$ is defined to refer to the reduced surface charge density using

$$
m=\frac{N_{t}}{L_{D} N_{D}} .
$$

The second part of the function given in 29 is the reduction, which involves several components. Essentially, the authors solve this in the same way as previously discussed, using the mass action law. For the different oxygen species $\mathrm{O}^{-}, \mathrm{O}_{2}^{-}$, and $\mathrm{O}^{2-}$ the derived equation for the exponent was

$$
\begin{gathered}
n=\frac{1}{2}\left(1-\frac{1}{m^{2}+1}\right), \\
n=1-\frac{1}{m^{2}+1}, \text { and } \\
n=\frac{1}{4}\left(1-\frac{1}{2 m^{2}+1}\right),
\end{gathered}
$$

respectively. The Equations 32,33, and 34 can be described by a single equation

$$
n=\frac{\alpha}{2 \beta}\left(1-\frac{1}{\beta m^{2}+1}\right),
$$

where $\alpha=1$ or $\alpha=2$ for dissociative or non-dissociative adsorption, respectively, and $\beta$ is the charge state of oxygen adsorbates on the surface. A power law exponent is furthermore calculated for the adsorption of reducing gases, and the adsorption of oxidizing gases. The calculated values seem to agree with the experiments shown; however, the full picture is also here not given. The subsequent adsorption of gas molecules to form a thin accumulation layer is not addressed and this is still a missing piece toward the development of a comprehensive model of the SMO sensing mechanism.

An additional aspect, which should be included when performing experiments on a SMO sensor, is the ability to accurately measure the temperature provided by the microheater in the active region. Temperature plays a significant role in defining the sensor signal, both the reception and transduction components; however, as it was discussed in an earlier section, the temperature uniformity and accurate measurements of the temperature are not easily obtained. In Ref. 97, the authors used several methods in order to attempt to extract the temperature, while to date no error-free method is known to the authors. In addition, humidity does not only influence the way in which the sensing proceeds, but it also changes the power dissipation of the SMO microheater. The change in power dissipation also changes the thermal resistive behavior of the microheater itself, effectively reducing the provided temperature for the active sensor area, ultimately influencing the sensing performance and introducing another layer of uncertainty. When developing SMO sensing behavior models based on experimental results, the accuracy of the experimental setup and proper extraction is of upmost importance. One way to ensure a proper link between the characterized and simulated temperatures is to avoid the complex microheater and membrane structure while performing the measurements, but instead using a heat chamber. This is most commonly done and it ensures the same conditions for the simulation and characterization. However, understanding how the sensor behaves when the temperature distribution across the SMO film is not uniform would be an important study in itself. In addition, including the influence of humidity on the change in the microheater temperature, and not only on the surface reactions, would go a long way in the formation of a fully-encompassed SMO simulator. Only through such a simulator, which covers multiple aspects of an SMO device, will a leap in the development and scaling of these devices be made possible.

Influence of noble metal additives.-As previously discussed, the introduction of noble metal additives has been used to increase the sensitivity and selectivity of SMO gas sensors. A perfect example is the use of $\mathrm{Pt}$ in $\mathrm{SnO}_{2}$ sensors for $\mathrm{CO}$ detection, shown in Figure 10d. Recently, researchers have attempted to address the influence of platinum additives in the reception-transduction behavior of the $\mathrm{SnO}_{2}$ sensor using operando spectroscopy. ${ }^{156}$ The authors found that nanosized clusters of Pt are formed on the sensing film's surface. These act as primary reaction sites for $\mathrm{CO}$ oxidation, thereby significantly increasing the sensing effect, when compared to pristine $\mathrm{SnO}_{2}$ films. The amount of platinum also determines whether $\mathrm{CO}$ oxidation or $\mathrm{CO}$ sensing will dominate, therefore simply having more of an additive does not automatically mean an improved sensor response, even if the additive is more reactive. This is clearly demonstrated in Ref. 87 and in Figure 10b, where the introduction of $0.2 \mathrm{wt} \% \mathrm{Pt}$ resulted in an improved performance, while increasing this to a $2 \mathrm{wt} \%$ reduced the sensing response to below pure $\mathrm{SnO}_{2}$ levels. A reproduction of these experiments in Ref. 139 in a humid environment showed that the response of the $2 \mathrm{wt} \%$ loaded film does not fall below that of the undoped film, but is nevertheless less sensitive than the $0.2 \mathrm{wt} \%$ alternative.

The ways in which a noble metal atom can influence the sensing film is described in some detail in several recent works ${ }^{156,157}$ and are partially summarized in Figure 22. The two primary mechanisms are chemical and electrical sensitization. The chemical sensitization mechanism is related to the spill-over of gases, which is primarily attributed to metallic clusters which adsorb oxygen or reducing gases. ${ }^{158}$ The adsorbed gas molecules are subsequently activated and transferred to the $\mathrm{SnO}_{2}$ surface, increasing the reactivity of the sensing film, thereby improving the sensitivity, as depicted in Figure 22a. The electrical sensitization mechanism is based on the Fermi level alignment of the SMO material and noble metal phase, due to the fact that the metal phase has a different work function. The contact between the two materials leads to an alignment of the Fermi levels and thereby surface band bending. This band bending is now controlled by the contact between the two materials and not by the presence of an ionosorbed species, depicted in Figure 22b. 


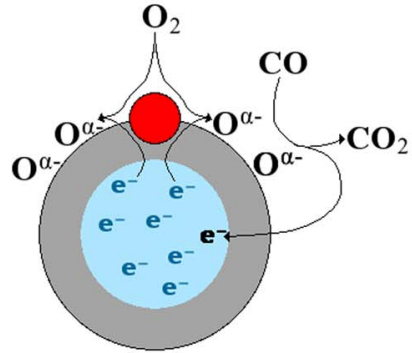

(a)

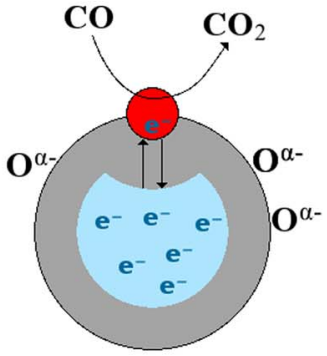

(b)

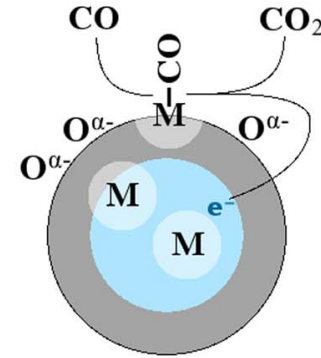

(c)

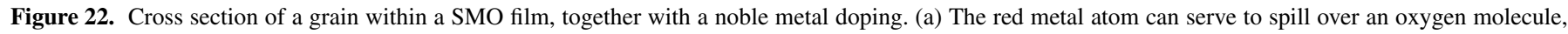

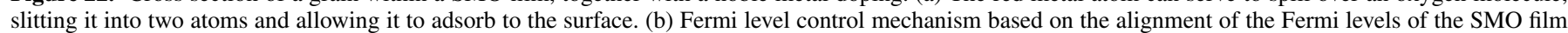

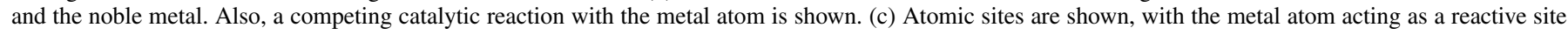
and possibly as an acceptor/donor. The images are adapted from Ref. 139.

In the absence of a chemical and electrical interaction between the metal phase and SMO film, a competitive reaction on the noble metal phase takes place, decreasing the concentration of the target gas, thereby hindering the sensing effect, shown in the top surface reaction in Figure 22b. ${ }^{139}$ However, an oxidation reaction at the interface of the two solids could enhance the gas reception and thereby the sensing behavior. ${ }^{157}$ The noble metal atoms or ions can also be incorporated in the SMO's lattice, changing the chemical and electrical behavior of the film and thereby the reception and transduction effects. In Figure $22 \mathrm{c}$ the various locations of noble metal atom incorporation in the SMO film and their effects are shown, as adapted from Ref. 139. If the valence state of the metal ion is different from that of the replaced cation, additional acceptor or donor states can be introduced. ${ }^{157}$ Particularly doping with $\mathrm{Pt}$ leads to a lowered resistance of the sensing film and an enhanced $\mathrm{CO}$ oxidation due to the increased number of oxygen vacancies generated. ${ }^{157,159}$

\section{Conclusions}

There has been significant progress recently in the design, modeling, and understanding of SMO gas sensors. These types of sensors are currently the cheapest and most portable option available for the detection of harmful environmental pollutants. The ability to integrate the device with CMOS circuitry and to fabricate it using a mature CMOS foundry are primary reasons for its success in industry and commercialization. However, in order to make a truly integrated smart gas sensor using an SMO film, there are several aspects which still need to be improved upon. Those include an improvement in the reliability of the different layers used as well as a complete understanding of the sensing mechanism itself, which is still lacking.

Advanced modeling and simulation tools have been used to obtain a deeper understanding of the fabrication, reliability, and operation of semiconductor metal oxide gas sensors. The advanced analysis and characterization have allowed us to recently develop optimized sensor designs which reduce the power consumption, improve temperature uniformity, and minimize stress accumulation. Of primary importance is reducing the power dissipation of gas sensor arrays to levels compatible with portable technologies, while ensuring proper temperature uniformity across the active sensor area. In this review, recent designs were compared for their power efficiency, showing that few large microheaters are preferred to many small ones. Recent designs introduce the concept of a microheater array, which takes advantage of this knowledge to ensure improved power dissipation while still allowing for multiple sensors in order to introduce selectivity to the SMO device. In addition, researchers are always searching for new materials, which have improved microheater properties; however, this is difficult as it is still desired to have these readily available in a CMOS fabrication facility, due to the associated cost reduction.

When characterizing the fabrication of SMO sensors, the essential processes are the etching step for the creation of a suspended membrane and the deposition of the sensing film itself. Both processing steps were analyzed using process simulation tools. For mechanical simulations, in order to understand the stress buildup and distribution in the complex structure, finite element analysis have been carried out. Electro-thermal characterization has been performed using FEM and an analytical model has been developed as a means to represent the thermal behavior of the sensor in an equivalent electrical circuit form. Using these simulations, we can perform a complete electro-thermalmechanical simulation to better understand the long-term mechanical reliability of the complex membrane structure of the SMO sensor. Recent designs have been described, which show the improved behavior of suspended membranes with rounded corners, due to the reduced current accumulation effects, which could lead to high stresses and eventual failure.

Finally, recent models which describe the sensing mechanism of SMO film through the reception-transduction behavior have been presented. Additional calculations have been performed in order to characterize the effects of gas ionosorption at the surface of SMO thin films. The adsorbed charges generate an effective surface potential, which forms an electric field, influencing the behavior of the charge carriers in the SMO and thereby also its conductance. While the conductivity of the SMO film can be described using drift diffusion equations with reasonable accuracy, the exact chemisorption steps are still not fully known. The most recent studies on the surface chemisorption of $\mathrm{CO}$ on $\mathrm{SnO}_{2}$ have been described in this review. While the general opinion was that the sensing mechanism involves the adsorption of oxygen at the surface, which creates a depletion layer, followed by a reaction of that oxygen with $\mathrm{CO}$ gas molecules to form $\mathrm{CO}_{2}$, thereby reducing the depletion layer once more. Although this is accurate in most cases, there are still events which are less clear, including the evidence of a sensing behavior even when little or no oxygen is present in the ambient or in an inert/CO ambient. The most recent understanding of these phenomena have been summarized in this review along with a discussion on the influence of noble metal additives to the SMO film. Primarily, the additives can improve the sensitivity, and sometimes selectivity, of the film; however, increasing the amount of additive metal does not immediate mean an increase in the sensitivity. There is a more complex relationship, which still requires further research to completely understand.

\section{ORCID}

Lado Filipovic (D) https://orcid.org/0000-0003-1687-5058 Ayoub Lahlalia (1D https://orcid.org/0000-0002-4680-0498

\section{References}

1. J.-H. Park et al., IEEE Sens. J., 17(3), 784 (2017).

2. D. Rüffer et al., Sensors, 18(4), 1052 (2018).

3. H. Li et al., IEEE Sens. J., 14(10), 3391 (2014).

4. R. Moos et al., Sens. Actuators B, Chem, 83(1-3), 181 (2002).

5. G. Martinelli et al., Sens. Actuators B, Chem, 55(2-3), 99 (1999). 
6. M. Carotta et al., Sens. Actuators B, Chem, 58(1-3), 310 (1999).

7. G. F. Fine et al., Sensors, 10(6), 5469 (2010).

8. A. A. Tomchenko et al., Sens. Actuators B, Chem, 108(1-2), 41 (2005).

9. F. Wang et al., J. Am. Chem. Soc., 130(16), 5392 (2008).

10. G. Neri, Chemosensors, 3(1), 1 (2015).

11. E. Singh et al., ACS Appl. Mater. Interfaces, 9(40), 34544 (2017).

12. E. Lee et al., ECS Meeting Abstracts, 42 (2018).

13. G. Errana et al., Crit. Rev. Solid State Mater. Sci., 29(3-4), 111 (2004).

14. G. Korotcenkov, Mat. Sci. Eng. B., 139(1), 1 (2007).

15. A. Dey, Mat. Sci. Eng. B., 229, 206 (2018)

16. X. Liu et al., Sensors, 12(7), 9635 (2012)

17. K. Arshak et al., Sensor Review, 24(2), 181 (2004).

18. O. Hotel et al., Sens. Actuators B, Chem, 255(3), 2472 (2018).

19. J. Devkota et al., Sensors, 17(4), 801 (2017).

20. M. Aleixandre and M. Gerboles, Chem. Eng. Trans., 30, 169 (2012).

21. S. Narayanan et al., Microchimica Acta, 181(5-6), 493 (2014).

22. A. S. Mustafaev et al., J. Phys.: Conf. Ser., 929, 012105 (2017).

23. S. O. Agbroko and J. Covington, Sens. Actuators B, Chem, 275, 10 (2018).

24. A. Ponzoni et al., Sensors, 17(4), 714 (2017).

25. D. Xu et al., Front. Mech. Eng., 12(4), 557 (2017).

26. AG ams, Ultra-Low Power Analog VOC Sensor for Indoor Air Quality Monitoring, 12 2016. CCS801: v1-02.

27. AG ams, Ultra-Low Power Gas Sensor for Ethanol Detection, 12 2016. CCS803: v1-00.

28. AG ams, Ultra-Low Power Digital Gas Sensor for Monitoring Indoor Air Quality, 12 2016. CCS811: v1-00.

29. Figaro USA, Inc. TGS 2600 - for the detection of Air Contaminants. TGS 2600.

30. Figaro USA, Inc. TGS 2602 - for the detection of Air Contaminants. TGS 2602.

31. Figaro USA, Inc. TGS 8100 - for the detection of Air Contaminants. TGS 8100.

32. B. T. Marquis and J. F. Vetelino, Sens. Actuators B: Chem, 77(1-2), 100 (2001).

33. Y. Mo et al., Sens. Actuators B: Chem, 79(2-3), 175 (2001).

34. N. Barsan et al., Sens. Actuators B: Chem, 121(1), 18 (2007).

35. K. T. Ng et al., IEEE Trans. Circuits Syst. I, 58(7), 1569 (2011).

36. T. Konduru et al., Sensors, 15(1), 1252 (2015).

37. L. A. Horsfall et al., J. Mater. Chem. A, 5(5), 2172 (2017).

38. N. Joshi et al., Microchimica Acta, 185(4), 213 (2018)

39. D. Zhang et al., Sens. Actuators B: Chem, 240, 55 (2017).

40. N. Barsan et al., Sens. Actuators B, Chem., 207(A), 455 (2015).

41. G. Korotcenkov et al., Solid State Phenom. 226, 187 (2017).

42. J. W. Gardner et al., IEEE Sens. J., 10(12), 1833 (2010).

43. J.-H. Suh et al., Sens. Actuators B, Chem, 265, 660 (2018).

44. E. Lackner et al., Mater. Today Proc., 4(7), 7128 (2017).

45. K. Banger et al., Nature Man., 10(1), 45 (2011).

46. A. Millar et al., J. Adv. Phys., 3(3), 179 (2014).

47. S. Park et al., Mat. Sci. Eng. R, 114, 1 (2017).

48. L. Znaidi, Mat. Sci. Eng. B, 174(1), 18 (2010).

49. I. Volintiru et al., Thin Solid Films 519(19), 6258 (2011).

50. Y. Lu et al., Vacuum 122, 347 (2015).

51. A. M. B. van Mol, Chemical Vapour Deposition of Tin Oxide Thin Films, PhD Thesis, Technische Universiteit Eindhoven (2003).

52. E. Boyalt et al., J. Alloys Compd., 692, 119 (2017).

53. S. Karthikeyan et al., Thin Solid Films, 550, 140 (2014).

54. K. M. Niang et al., Phys. Status Solidi A, 214(2), 122109 (2017).

55. J. Montero et al., J. Vac. Sci. Technol. B Nanotechnol. Microelectron. 33(3), Article ID 031805 (2015).

56. R. Kukla, Surf. Coat. Technol., 93(1), 1 (1997).

57. G. Korotcenkov and B. Cho, Prog. Cryst. Growth Charact. Mater., 63(1), pp. 1 (2017).

58. G. Korotcenkov et al., Sens. Actuators B, Chem, 77(1), 244 (2001)

59. T. Hyodo et al., Sens. Actuators B, Chem, 244, 992 (2017).

60. A. Chen et al., Surf. Coat. Technol., 322, 120 (2017).

61. D. Perednis and L. Gauckler, Solid State Ionic, 166(3-4), 229 (2004)

62. L. Filipovic et al., IEEE Trans. Semicond. Manuf, 27, 269 (2014).

63. G. Korotcenkov et al., Mater. Sci. Eng., B, 77(1), 33 (2000).

64. S. Sinha et al., Mat. Lett., 65(2), 146 (2011).

65. M. Kwoka and M. Krzywiecki, Mat. Lett., 154, 1 (2015).

66. I. Simon et al., Sens. Actuators B, Chem., 73(1), 1 (2001).

67. P. Bhattacharyya, IEEE Tran. Dev. Mat. Reliab., 14(2), 589 (2014).

68. L. Filipovic and S. Selberherr, ECS Transactions, 85(8), 151 (2018).

69. Q. Zhou et al., Sens. Actuators A, Phys., 223, 67 (2012).

70. M. Prasad et al., J. Micro. Nanolithogr. MEMS MOEMS 14(2), Article ID 025003 (2015).

71. B.-U. Moon et al., Sens. Actuators B, Chem., 108(1), 271 (2005).

72. L. Filipovic and S. Selberherr, in Proc. TENCON 2015, 1 (2015).

73. M. Prasad, Microelectron.Rel., 55(6), 937 (2015).

74. S. Z. Ali et al., J. Microelectromech. Sys., 17(6), 1408 (2008).

75. R. Speer et al., Patent publication number US9164052B1 (2015).

76. R. Shankar et al., Patent publication number US20160018356 A1 (2016).

77. O. Le Neel et al., Patent publication number US20180156747 A1 (2018).

78. A. Lahlalia et al., IEEE Sens. J., 18(5), 1960 (2018).

79. W. H. Brattain and J. Bardeen, Bell Sys. Tech. J. 32(1), 1 (1953).

80. T. Seiyama et al., Anal. Chem., 34(11), 1502 (1962).

81. P. J. Shaver, Appl. Phys. Lett., 11(8), 255 (1967).

82. N. Taguchi, "Gas-detecting device", US Patent No. 3,631,436 (1971)

83. N. Taguchi, "Gas detecting device", US Patent No. 3,695,848 (1972).
84. G. Velmathi et al., IETE Tech. Rev. 33(3), 323 (2016).

85. A. Galdikas et al., Sens. Actuators B, Chem. 48(1-3), 376 (1998)

86. R. Wimmer-Teubenbacher et al., Mater. Today Proc., 2(8), 4295 (2015).

87. L. Mädler et al., J. Nanopart. Res., 8(6), 783 (2006).

88. L. Filipovic and S. Selberherr, Sensors, 15(4), 1843 (2015).

89. V. K. K. Tangirala et al., Sensors, 17(5), 1011 (2017).

90. A. Harley-Trochimczyk et al., J. Micromech. Microeng. 27(4), Article ID 045003 (2017).

91. M. Y. Afridi et al., IEEE Sens. J. 2(6), 644 (2002).

92. Q. Zhou et al., Sens. Actuators A, Phys., 223, 67 (2015).

93. L. Mele et al., Sens. Actuators A, Phys., 188, 173 (2012).

94. L. L. R. Rao et al., Proc. ICSTM, 624 (2015).

95. S. Bedoui et al., Proc. $13^{\text {th }}$ SSD, 558 (2016).

96. Y. Li et al., AIP Advances 4(3), Article ID 031339 (2014).

97. A. Lahlalia et al., J. Microelectromech. Sys. 27(3), 529 (2018).

98. R. Phatthanakun et al., Proc. ECTI-CON, Paper ID 1034, 14 (2011).

99. K. Zhang et al., Int. J. Therm. Sci., 46(6), 580 (2007).

100. L. Xu et al., IEEE Sens. J., 11(4), 913 (2011).

101. U. Dibbern, Sens. Actuator B, Chem., 2(1), 63 (1990).

102. W. Yan et al., J. Alloy Compd., 449(1-2), 210 (2008).

103. D. Monika and A. Arora, J. Adv. Res. Technol. 2(8), 2487 (2013).

104. S. M. Lee et al., Microelectron. J. 34(2), 115 (2003).

105. R. G. Spruit et al., J. Microelectromech. Sys., 26(6), 1165 (2017).

106. S. Astié et al., Sens. Actuators B, Chem. 67(1), 84 (2000).

107. F. Udrea et al., Sens. Actuators B, Chem. 78(1-3), 180 (2001)

108. D. Briand et al., J. Micromech. Microeng. 12(6), 971 (2002).

109. Y. Mo et al., Sens. Actuator A, Phys. 100(1), 94 (2002).

110. C. Tsamis et al., Sens. Actuator B, Chem. 95(1-3), 78 (2003).

111. J. Laconte et al., IEEE Sens. J. 4(5), 670 (2004)

112. T. Iwaki et al., J. Phys. Conf. Ser 15, 27 (2005).

113. M. Graf et al., Sens. Actuator B, Chem. 117(2), 346 (2006).

114. U. Frey et al., IEEE J. Solid-State Circuit 42(2), 441 (2007).

115. S. Roy et al., Proc. IECR, 59 (2010).

116. M. Y. Afridi and J. Geist, Proc. IEEE Sensors, 2159 (2010).

117. M. Prasad et al., Sens. Rev. 32(1), 59 (2012).

118. M. Siegele et al., Proc. 11th NEWCAS (2013).

119. N. André et al., Proc. ICICDT (2015).

120. V. Avramescu et al., Proc. ESSDERC, 280 (2016).

121. P. B. Deo, J. Technol. Adv. Sci. Res., 2(1), 79 (2016)

122. N. Yamazoe and N. Miura, Chem. Sens. Technol., 4, 19 (1992).

123. Y. Mo et al., Sens. Actuator B, Chem., 79(2-3), 175 (2001).

124. M. H. Saberi et al., Sens. Actuator B, Chem., 206, 617 (2015).

125. S. Banerjee, A. Dan, and D. Chakravorty, J. Mater. Sci., 37(20), 4261 (2002)

126. Y. Cui et al., Science, 293(5533), 1289 (2001).

127. I. Elmi et al., Sens. Actuators B, Chem., 135(1), 342 (2008)

128. L. Filipovic and S. Selberherr, IEEE Trans. Device Mater. Rel. 16(4), 483 (2016)

129. A. Götz et al., Sens. Actuators B, Chem. 44(1), 483 (1997).

130. B. Radjenović and M. Radmilović-Radjenović, Thin Solid Films 517(14), 4233 (2009).

131. ViennaTS. The Vienna Topography Simulator online, http://www.iue.tuwien.ac.at/ software/software/viennats/.

132. S. Gomez et al., J. Vac. Sci. Technol., B, 22, 606 (2004).

133. R. J. Belen et al., J. Vac. Sci. Technol., A, 23(1), 99 (2005).

134. L. Filipovic and S. Selberherr, Microelectron. Rel. 61, 3 (2016).

135. L. Filipovic et al., Microelectron. Eng. 107, 23 (2014).

136. S. C. Seel, Stress and Structure Evolution During Volmer-Weber Growth of Thin Films, PhD Dissertation, Massachusetts Institute of Technology (2002).

137. H. D. Baehr and K. Stephan, Wärme- und Stoffübertragung, $3^{\text {rd }}$ ed. Berlin, Germany; Springer 1998

138. L. Filipovic and S. Selberherr, ECS Transactions 66(5), 243 (2015).

139. D. Degler, Spectroscopic Insights in the Gas Detection Mechanism of Tin Dioxide Based Gas Sensors, PhD Thesis, Eberhard Karls Universität Tübingen (2017).

140. M. Batzill and U. Diebold, Prog. Surf. Sci. 79(2-4), 47 (2005).

141. Z. M. Jarzebski and J. P. Morton, J. Electrochem. Soc. 123(10), 333C (1976)

142. G. Tulzer et al., Nanotechnol. 24(31), 315501 (2013).

143. A. Fort et al., Sens. Actuators B, Chem. 124(1), 245 (2007).

144. N. Barsan and U. Weimar, J. Electroceramics 7(3), 143 (2001).

145. N. Barsan and U. Weimar, Proc. IMCS, 618 (2012).

146. A. Gurlo, Chemphyschem., 7(10), 2041 (2006).

147. D. Degler et al., J. Phys. Chem. C, 119(21), 11792 (2015).

148. N. Barsan et al., Sens. Actuator B, Chem, 157(2), 510 (2011)

149. J.-M. Ducéré et al., J. Comput. Chem., 33(3), 247 (2012).

150. X. Wang et al., J. Chem. Phys., 118(49), 28548 (2014).

151. S Wicker, Influence of Humidity on the Gas Sensing Characteristics of $\mathrm{SnO}_{2}-$ DRIFTS Investigation of Different Base Materials and Dopants, PhD Thesis, Eberhard Karls Universität Tübingen (2016).

152. Z. Hua et al., Sens. Actuator B, Chem., 255(2), 1911 (2018).

153. Z. Hua et al., Sens. Actuator B, Chem., 255(3), 3541 (2018).

154. Z. Hua et al., Sens. Actuator B, Chem., 267, 510 (2018).

155. G. Chabanis et al. Meas. Sci. Technol., 14(1), 76 (2003).

156. D. Delger et al., J. Mater. Chem. A, 6, 2034 (2018).

157. S. A. Müller et al. ChemCatChem, 10(5), 864 (2018).

158. M. Hübner et al. Phys. Chem. Chem., 14(38), 13249 (2012).

159. G. N. Vayssilov et al., J. Phys. Chem. C, 115(47), 23435 (2011). 